\title{
Algae Diversity and Ecology during a Summer Assessment of Water Quality in the Abraham Lincoln Birthplace National Historical Park, USA
}

\author{
Sophia Barinova ${ }^{1, *(\mathbb{D})}$ and Thomas Smith ${ }^{2}$ \\ 1 Institute of Evolution, University of Haifa, Abba Khoushi Ave, 199, Mount Carmel, Haifa 3498838, Israel \\ 2 Arkansas State University Beebe, 1000 W Iowa St, Beebe, AR 72012, USA; TESmith@asub.edu \\ * Correspondence: sophia@evo.haifa.ac.il; Tel.: +972-4824-97-99
}

Received: 5 September 2019; Accepted: 25 October 2019; Published: 1 November 2019

check for updates

\begin{abstract}
There were 88 species of algae and cyanobacteria observed from seven sites in the Abraham Lincoln Birthplace National Historical Park (Hodgenville, Kentucky, USA). This was the first algal investigation study in the park. There were 21 samples collected, during the summer, on 16 July 2008. Algal flora, dominated by diatoms was represented by 54 species identified (61.4\% of the total), 20 species of cyanobacteria, 11 green and two charophyte algal species, and one red algal species $(22.7 \%, 12.5 \%, 2.2 \%$, and $1.1 \%$, respectively). Benthic diatoms dominated the aquatic system with 14 species of Navicula and 12 species of Nitzschia identified, which was $15.7 \%$ and $13.5 \%$ of the total, respectively. Species tended to be site specific and $78.6 \%$ of the species were only found in two or less sites. The bioindicator methods for water quality assessment were based on species autoecology. This method was used for the first time in the USA during this study. This demonstrated that benthic and planktonic-benthic algae preferred temperate temperatures, middle-oxygenated mesotrophic waters, low-to-middle enriched by chlorides. The waters were well oxygenated, sometimes saturated by sulfides, low-alkaline, low-to-middle organic enriched, and of class 1-3 water quality with high self-purification capacity. This is very important for habitat protection and cannot be easily accomplished strictly through chemical analysis. The diversity of diatom algae not only plays a major role in the formation of algal communities and their uniqueness, but diatom algae can be a good indicator of environmental assessments and change.
\end{abstract}

Keywords: freshwater algae; diversity; ecology; bioindicator; water quality; protected area; Abraham Lincoln Birthplace National Historical Park

\section{Introduction}

Abraham Lincoln Birthplace National Historical Park (ALBNHP) is located in Kentucky, USA and has a total area of 38.8 hectares. The park is part of the Knobs Region. This region has a mixed hardwood forest with a predominance of oak and hickory trees [1]. The ALBNHP receives an average of $128 \mathrm{~cm}$ of rainfall each year which varies little throughout the year, while the average temperatures range from $19.7^{\circ} \mathrm{C}$ in the summer to $7.6^{\circ} \mathrm{C}$ in the winter. The bedrock is dominated by limestone with a sandstone cap. The region has a karst topography with many sinkholes. There is very little surface water existing in the park due to karst topography [1].

There are 606 vascular plants identified from the ALBNHP, but studies of algae and cyanobacteria are lacking. In fact, no studies have investigated algae within the park. This is the first phycological study in ALBNHP. There have been other algal studies conducted in Kentucky from caves [2-4], terrestrial [5], a few from lotic [6-10] and lentic [11-14] habitats and general statewide surveys [15-19].

Floristic studies in aquatic systems are very important because the flora are environmental indicators [20] which infer environmental conditions on the natural, climatic, and economic from 
the Knob Region. At the same time, its territory is replete with a variety of lotic and lentic water bodies with especially ephemeral streams because of the karst topography. Aquatic studies of algae are of special interest since the formation of their flora and algal composition is strongly influenced by land-water interactions [20]. Thus, the algal list represents the combined result of natural and anthropogenic processes in the entire catchment area for many years.

The aim of this study is to evaluate the current ecological condition of the waterbodies from the Abraham Lincoln Birthplace National Historical Park, based on the freshwater algae taxa composition and ecology with bioindicators through statistical methods.

\section{Materials and Methods}

The study of the algae in the waterbodies of the Abraham Lincoln Birthplace National Historical Park was carried out on material collected, during the summer, on 6 July 2008. This was a one-time sampling collection. The samples collected came from 7 sites and 4 different waterbodies with a total of 21 samples (3 replicate samples per site).

Samples were taken in the littoral and pelagial in plankton, benthos, and epilithic from stones. Phytoplankton was collected with an Apstein net and stored in sterile bottles. The epilithic rock samples were cleaned with a soft toothbrush by lightly scrubbing in a circular motion, then rinsed with distilled water. The toothbrush was cleaned with distilled water after each sample. This rinse water was collected in a sterile Whirl-pak. Phytobenthos samples were collected with a large nylon liquid baster and placed in a sterile Whirl-pak. Algal and water samples were transported to the lab in an ice cooler at $0{ }^{\circ} \mathrm{C}$ until they could be taken to the lab for processing.

The temperature, conductivity, and $\mathrm{pH}$ of the water were measured at the time of sampling with a HANNA Waterproof Portable pH/Temperature meter and an Oakton Waterproof CON 150 Meter. There were 21 water samples analyzed in the lab with a Shimadzu ICPE-9000 multitype inductively coupled plasma (ICP) emission spectrometer for $\mathrm{Hg}$, $\mathrm{TP}$, and $\mathrm{NO}_{3}$.

Diatom slides were prepared with a peroxide technique [21]. All slides of soft and diatom algae were identified with a Leica DM2500 light microscope under 400 to 1000x magnification.

International handbooks were used to identify algae [22-39]. To update the nomenclature, we used the Algaebase.org website [40]. Therefore, all names are currently accepted taxonomically.

The determination of saprobic indices was carried out according to the Pantle-Buck method in Sládeček's modification [19].

Saprobity indices were obtained for each algal community as a function of the number of saprobic species and their relative abundances:

$$
S=\sum_{i=1}^{n}\left(s_{i} h_{i}\right) / \sum_{i=1}^{n}\left(h_{i}\right),
$$

where $S$ is the index of saprobity for algal community (unitless), $s$ is the species-specific saprobity index, and $n$ is the cell density of each species.

The similarity calculations were performed using the BioDiversity Pro 2.0 program and network analyses in JASP were performed using the botnet package in R Statistica package of [41]. The canonical correspondence analyses were done with the CANOCO Program 4.0 [42]. The program GRAPHS [43] was used for comparative floristic of revealed communities. The bioindicator analyses were done with the help of ecological preferences of identified algae and cyanobacteria species [44-46].

\section{Description of Study Sites}

The Abraham Lincoln Birthplace National Historical Park study site is located in the Knob Region (Figure 1) of Kentucky, USA. The ALBNHP has two park locations (site A and site B). They are roughly $12 \mathrm{~km}$ apart (Figure 2). Site A had only one sampling location (sample \#1), which is the Sinking Spring (Figure 3a). The Sinking Spring is groundwater recharged and flows between layers of the limestone 
bedrock. Site B had sampling locations 2 to 7 (Figure 3b, Table 1). Sample \#2 was plankton towed from an artificial pond (Figure 4b), whereas samples \#3 to \#7 were from ephemeral streams (Figure 4a, Figure 5a,b and Figure 6a,b). At sample location \#5, the stream was sinking into the bedrock (Figure 6a) and at sample location \#6, the stream was reemerging (Figure 6b).

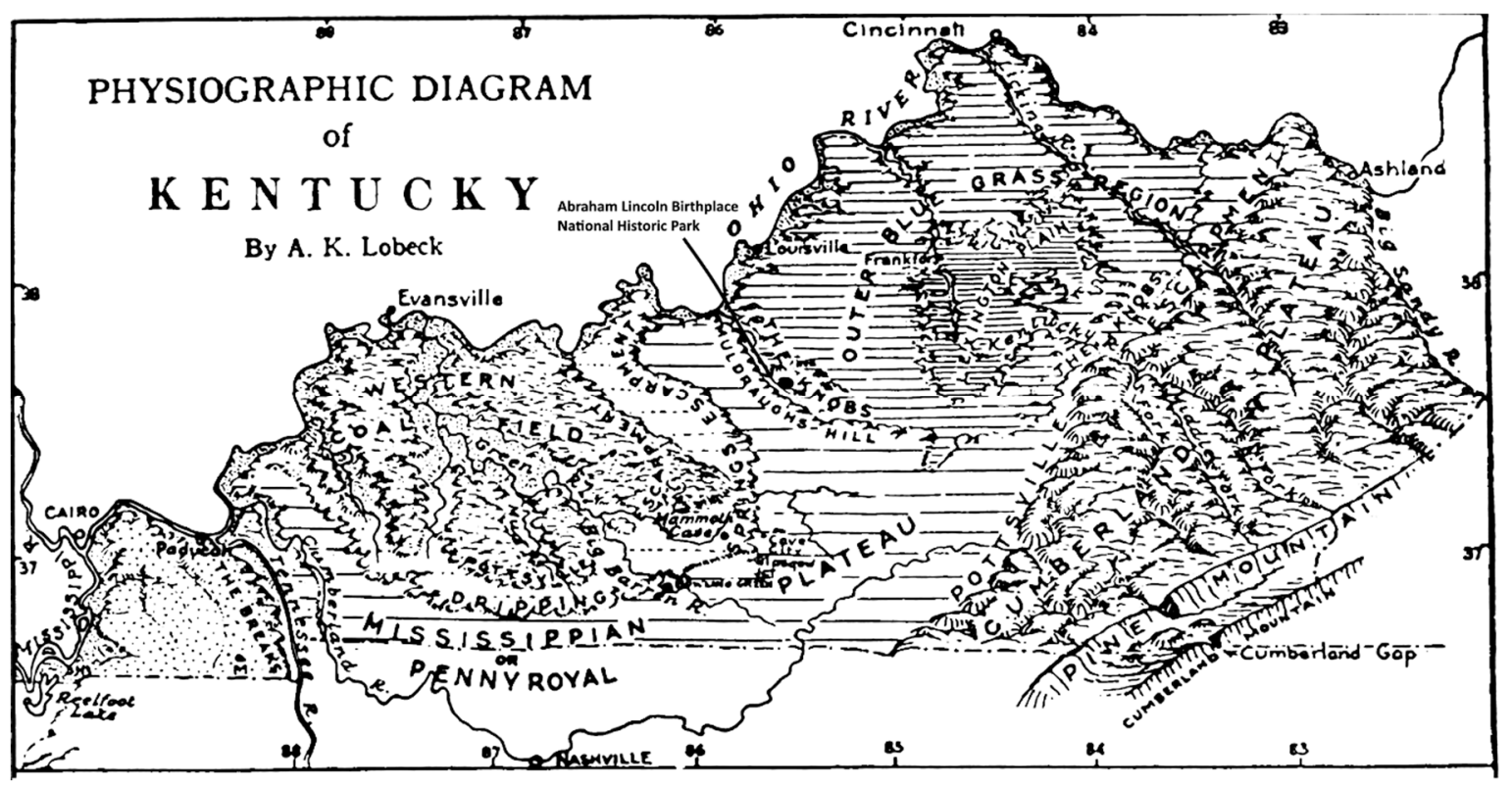

Figure 1. Location of Abraham Lincoln Birthplace National Historical Park in Kentucky, USA from Lobeck 1930.

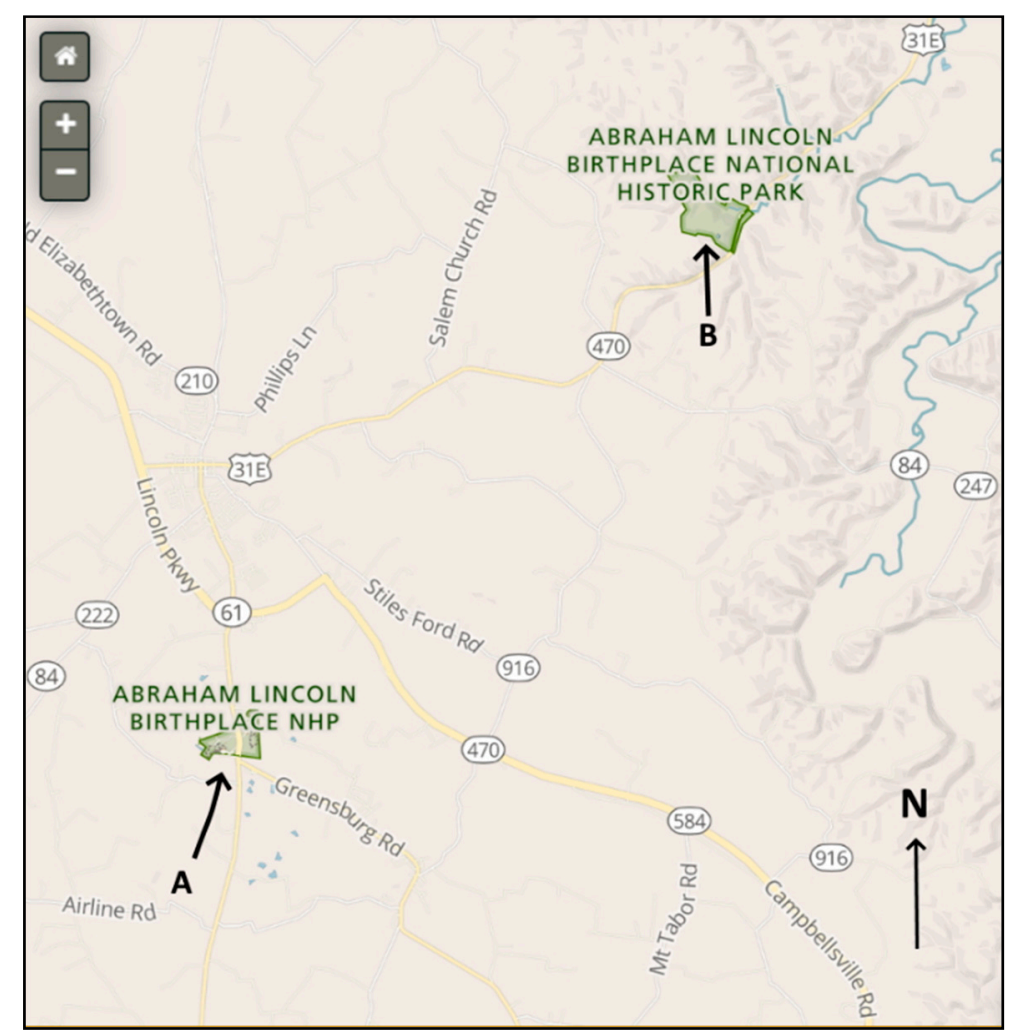

Figure 2. Location of the two sampling locations in Abraham Lincoln Birthplace National Historical Park in Kentucky, USA. 

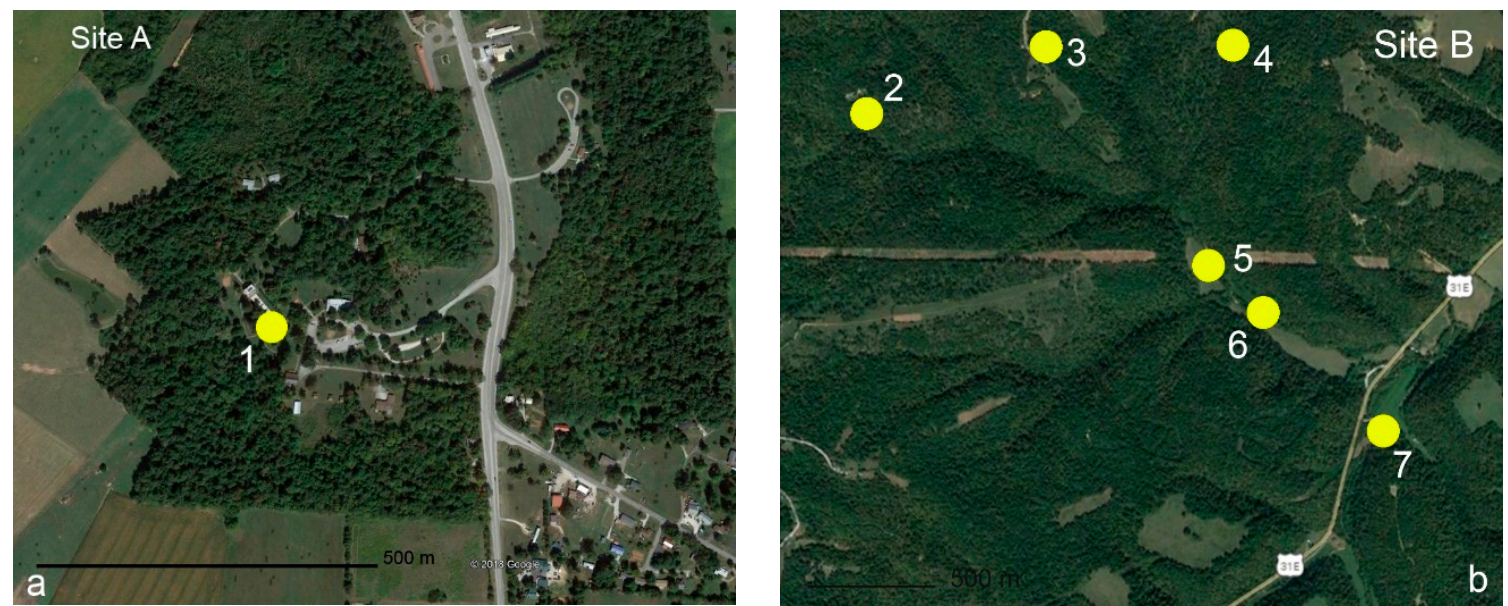

Figure 3. Location of the sampling site A (a) and site B (b) for samples \#1 to \#7.

Table 1. Site (\#1-7) and samples descriptions from the Abraham Lincoln Birthplace National Historical Park in Kentucky, USA.

\begin{tabular}{|c|c|c|}
\hline Site & Sample & Site Description \\
\hline A & 1 & $\begin{array}{l}\text { "Sinking Spring"—groundwater flow out of bedrock. Samples moss } \\
\text { and diatoms on wet sediments }\end{array}$ \\
\hline B & 2 & Artificial Pond-plankton tow. Whole water samples \\
\hline B & 3 & $\begin{array}{l}\text { Ephemeral stream-small pools and little water flow. Open canopy. } \\
\text { Epilithic, Epiphytic samples }\end{array}$ \\
\hline B & 4 & $\begin{array}{l}\text { Ephemeral stream-small riffles and little water flow. Closed canopy. } \\
\text { Epilithic, Epiphytic samples }\end{array}$ \\
\hline B & 5 & $\begin{array}{l}\text { Sinking stream-small flowing pool. Closed canopy. Epilithic, } \\
\text { Epiphytic samples }\end{array}$ \\
\hline B & 6 & $\begin{array}{l}\text { Emerging stream—small flowing pool. Closed canopy. Epilithic, } \\
\text { Epiphytic samples }\end{array}$ \\
\hline B & 7 & $\begin{array}{l}\text { Knob Creek—stream—small pools and little water flow. Open canopy. } \\
\text { Epilithic, Epiphytic samples }\end{array}$ \\
\hline
\end{tabular}
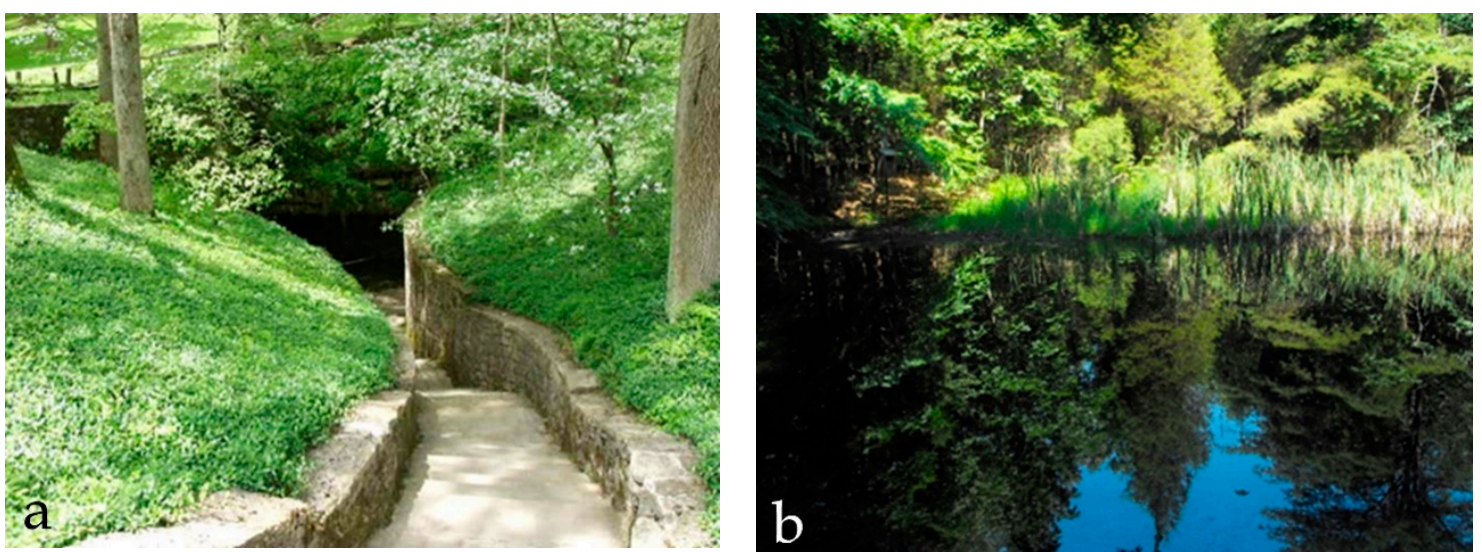

Figure 4. The sampling locations \#1 (a) and \#2 (b) from the Abraham Lincoln Birthplace National Historical Park in Kentucky, USA. 

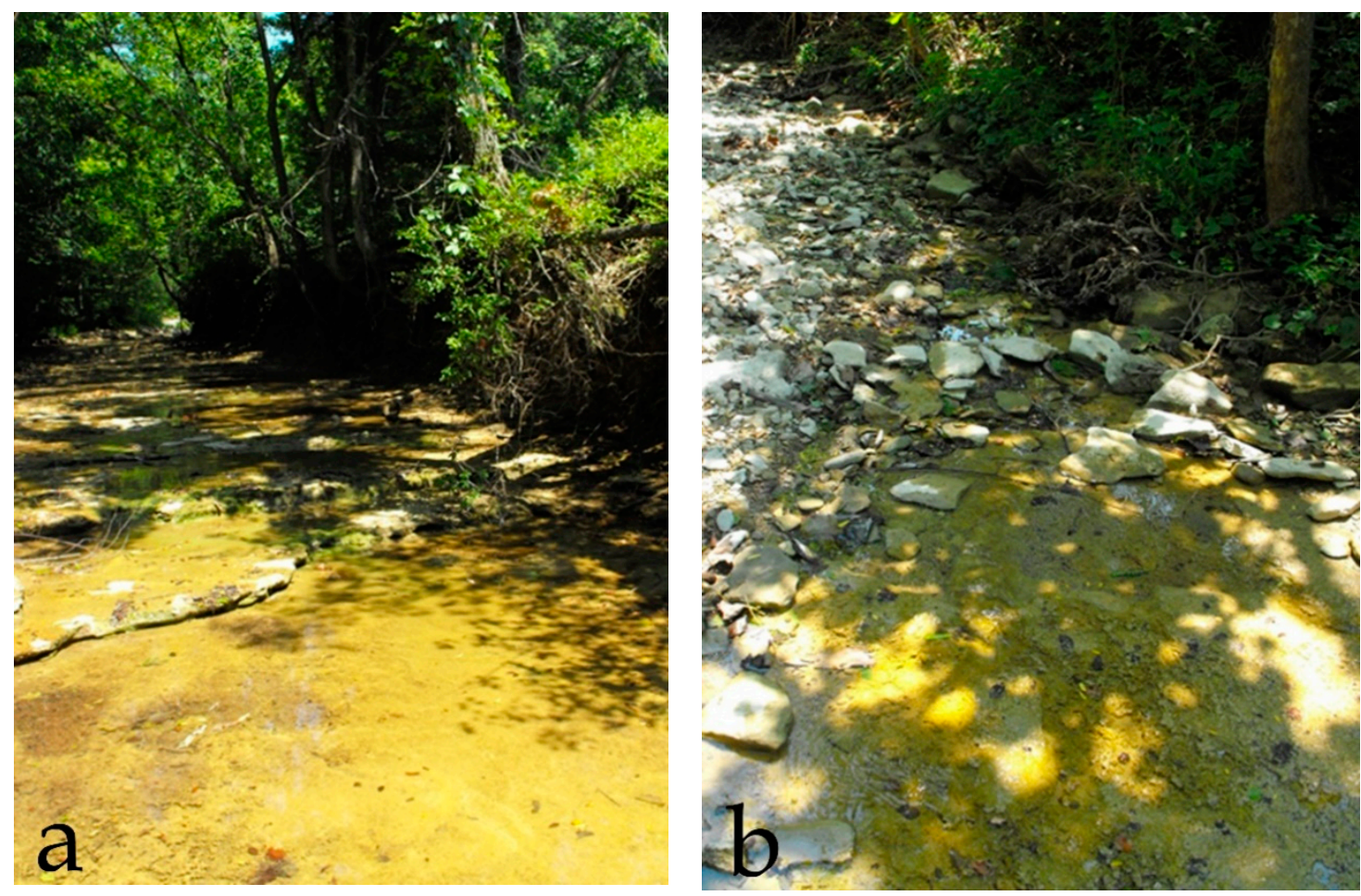

Figure 5. The sampling locations \#3 (a) and \#4 (b) from the Abraham Lincoln Birthplace National Historical Park in Kentucky, USA.
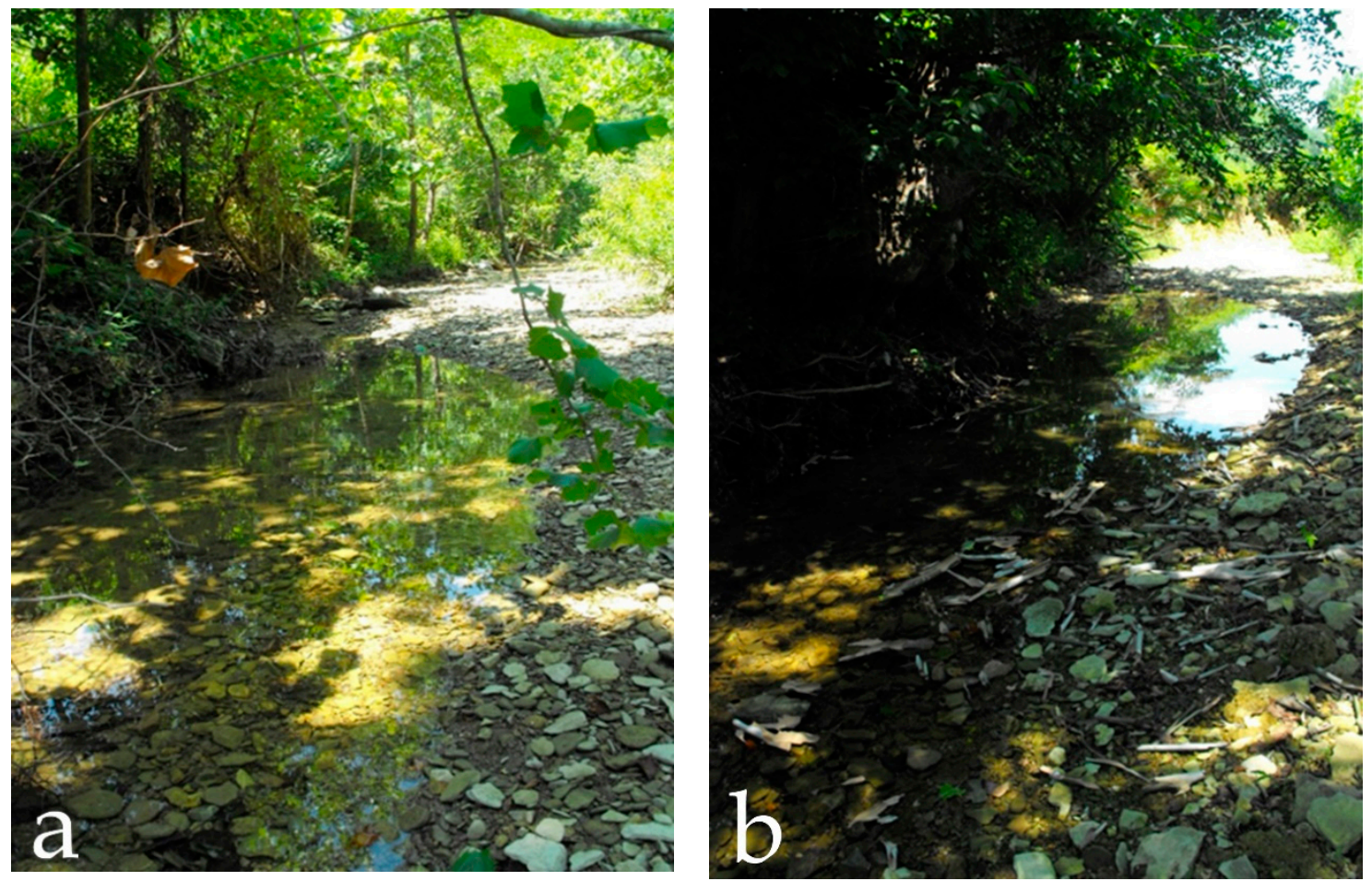

Figure 6. The sampling locations \#5 (a) and \#6 (b) from the Abraham Lincoln Birthplace National Historical Park in Kentucky, USA.

\section{Results}

The water chemistry and geographical position of seven studied sites are represented in Table 2. The elevations of the studied waterbodies are very similar; they are located at 137-260 m a.s.1. 
The average water temperature during the sampling period varied between $14.2^{\circ} \mathrm{C}$ from the cave wall and in the small pool of site \#1 and $26^{\circ} \mathrm{C}$ in the sinking stream of site \#5. Water conductivity and total dissolved solids (TDS) have minimal values in the pond of site \#3, whereas the maximal values are from sites \#1 and \#3. The $\mathrm{pH}$ of the water $\mathrm{pH}$ ranged with an amplitude of 7.4 (site \#1) and 8.3 (site \#4), with all sites being alkaline. Total phosphorus (TP) and nitrate values were minimal but the quantity available was enough for base growth. Only one heavy metal, $\mathrm{Hg}$, was determined in this study. The Hg metal had a minimal value at site \#7 (13 ppm), but maximal value at site \#2 (40 ppm) where water conductivity and TDS had the lowest values.

Table 2. Averaged environmental data of the sampling stations in the Abraham Lincoln Birthplace National Historical Park with its geographical position.

\begin{tabular}{ccccccccccc}
\hline Site & $\begin{array}{c}\mathbf{H g} \\
(\mathbf{p p m})\end{array}$ & $\begin{array}{c}\mathbf{T P} \\
(\mathbf{p p m})\end{array}$ & $\begin{array}{c}\mathbf{N O}_{3} \\
(\mathbf{p p m})\end{array}$ & $\begin{array}{c}\mathbf{T} \\
\left({ }^{\circ} \mathbf{C}\right)\end{array}$ & $\mathbf{p H}$ & $\begin{array}{c}\text { Conductivity } \\
\left(\mu \mathbf{~ c m}^{-1}\right)\end{array}$ & $\begin{array}{c}\text { TDS } \\
(\mathbf{p p m})\end{array}$ & $\begin{array}{c}\text { Elevation } \\
(\mathbf{m})\end{array}$ & Latitude & Longitude \\
\hline 1 & 36 & $<0.3$ & 0.89 & 14.2 & 7.5 & 460 & 230 & 261 & 37.530 & -85.74 \\
2 & 40 & $<0.3$ & 0.83 & 22 & 7.4 & 30 & 10 & 241 & 37.608 & -85.64 \\
3 & 16 & $<0.3$ & 0.15 & 20 & 7.7 & 450 & 230 & 189 & 37.614 & -85.65 \\
4 & 23 & $<0.3$ & 0.58 & 25 & 8.3 & 350 & 170 & 167 & 37.614 & -85.64 \\
5 & 20 & $<0.3$ & 0.64 & 26 & 8.2 & 340 & 170 & 180 & 37.613 & -85.64 \\
6 & 17 & $<0.3$ & 0.72 & 22 & 8.1 & 320 & 160 & 178 & 37.613 & -85.64 \\
7 & 13 & $<0.3$ & 1.41 & 19 & 7.7 & 410 & 210 & 137 & 37.611 & -85.64 \\
\hline
\end{tabular}

Note, the number of studied waterbodies sites as: 1 , cave wall and small pool; 2 , pond; 3 , stream and pool; 4 , stream and riffle; 5, stream (sinking), 6, stream (emerging); 7, Knob Creek ephemeral stream.

There were 88 species of algae and cyanobacteria observed in the waterbodies from seven study sites of the Abraham Lincoln Birthplace National Historical Park (Table 3). Diatoms were strongly represented with 54 species, followed by cyanobacteria with 20 species, and green algae with 11 species. Charophyta and Rhodophyta divisions were represented by two and one species, respectively. The distribution of taxonomical content in each site is represented in Table 3 . This is the first algal diversity study from the ALBNHP. We analyzed the taxonomical group's distribution and diversity, as well as species ecological preferences according to [44,45], which is listed in Table 3. It can be seen that the species richness was highest on the cave wall and in the small pool of site \#1 (36) and in the Knob Creek ephemeral stream of site \#7 (35), whereas minimal species richness was revealed in the stream and pool of site \#3 (13). Diatoms prevailed in all the studied communities but in site \#7 it was accompanied by twenty cyanobacteria species. Figure 7a demonstrates that in sites \#3 to \#7, the cyanobacteria species also played an important role in algal communities where they contained up to $10 \%$ to $15 \%$ of total species richness in the site's community. Porphyridium purpureum (Rhodophyta) algae was represented in sites \#2, \#3, and \#7.

Bioindicator plots of algal species preferences for each site community are shown in Figures 7-11 and Tables 3 and 4. Therefore, algae in the studied habitats of the Abraham Lincoln Birthplace National Historical Park have mostly benthic and planktonic-benthic ecology with up to $8 \%$ to $15 \%$ of planktonic species, and $3 \%$ to $8 \%$ of epiphytes and soil inhabitants (Figure $7 \mathrm{~b}$ ).

Temperature indicators were represented by four ecological groups (Figure 8a). Temperate temperatures of water were preferred by most species, cool-water indicators in sites \#1 and \#2 represented up to $23 \%$ of species richness of these communities, and warm temperature regimes were preferred by $20 \%$ of species in site \#7. Lotic and oxygen saturation indicators (Figure $8 \mathrm{~b}$ ) could be characterized from sites \#1 to \#5. They were well oxygenated, but, in sites \#6 and \#7, species were found that could survive in waters enriched by sulfides, as a result of organic degrading destruction in low moving water. The aerophilic community species were observed in the stream and pool site \#3 that demonstrates a high-oxygen enrichment environment. 
Table 3. Diversity of algae in the Abraham Lincoln Birthplace National Historical Park habitats with the species-specific ecology. Sampling points are given as 1-7 as in Tables 1 and 2 .

\begin{tabular}{|c|c|c|c|c|c|c|c|c|c|c|c|c|c|c|c|c|c|c|}
\hline Taxa & Code & 1 & 2 & 3 & 4 & 5 & 6 & 7 & Hab & Temp & Rheo & $\mathrm{pH}$ & Sal & Wat & Tro & Aut-Het & Sap & Index S \\
\hline \multicolumn{19}{|l|}{ Cyanobacteria } \\
\hline $\begin{array}{l}\text { Aphanocapssa grevillei (Berkeley) Rabenhorst } \\
\text { Aphonocass }\end{array}$ & $\begin{array}{l}\text { AphGre } \\
\text { Anhivi }\end{array}$ & $0_{0}^{0}$ & 0 & 0 & 0 & 0 & 0 & 1 & $\mathrm{P}$ & - & - & - & $\mathrm{hb}$ & - & - & - & $\mathrm{o-b}$ & 1.4 \\
\hline $\begin{array}{l}\text { Aphanocapsa rivularis (Carmichael) Rabenhorst } \\
\text { Chroococcus chenerens (Brébisson) Nägeli }\end{array}$ & $\begin{array}{l}\text { AphRivi } \\
\text { ChrCh }\end{array}$ & ${ }_{0}^{0}$ & $\begin{array}{l}0 \\
0\end{array}$ & ${ }_{0}^{0}$ & ${ }_{0}^{1}$ & ${ }_{0}^{0}$ & ${ }_{0}^{0}$ & 0 & $\begin{array}{c}\mathrm{Ep}, \mathrm{S} \\
\mathrm{BS}\end{array}$ & - & - & - & $\mathrm{hb}$ & - & o & - & $\mathrm{o}$ & 1.3 \\
\hline $\begin{array}{l}\text { Chroococccus cohaerenss (Brebisson) Nägeli } \\
\text { Chrococcus varius A.Braun }\end{array}$ & $\begin{array}{l}\text { ChrCoh } \\
\text { ChrVar }\end{array}$ & ${ }_{0}^{0}$ & ${ }_{0}^{0}$ & 0 & ${ }_{0}^{0}$ & ${ }_{0}^{0}$ & 0 & 1 & B,S & - & aer & - & $\mathrm{hb}$ & - & - & - & - & 1 \\
\hline $\begin{array}{l}\text { Chroococcus voriulu A.Braun } \\
\text { Cyanobium plancticum (G.Drews, H.Prauser \& D.Uhlmann) Komárek, Kopeckł \& Cepák }\end{array}$ & $\begin{array}{l}\text { ChrVar } \\
\text { CyaPla }\end{array}$ & ${ }_{0}^{0}$ & $\begin{array}{l}0 \\
0\end{array}$ & ${ }_{0}^{0}$ & ${ }_{1}^{0}$ & ${ }_{1}^{0}$ & ${ }_{0}^{0}$ & $\frac{1}{0}$ & $\mathrm{~B}_{\mathrm{P}}^{\mathrm{B}} \mathrm{S}$ & - & aer & - & - & :- & he & - & ${ }^{o-b}$ & 1.4 \\
\hline 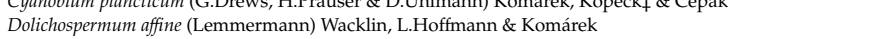 & $\begin{array}{l}\text { Yalia } \\
\text { DolAff }\end{array}$ & 1 & 0 & 0 & ${ }_{0}^{1}$ & ${ }_{0}^{1}$ & 0 & 0 & ${ }_{P}^{P}$ & - & - & $=$ & - & - & he & - & $=-a$ & 1.85 \\
\hline Gloeocapsa granosa (Berkeley) Kützing & GloGra & 0 & 1 & 0 & 1 & 0 & 0 & 0 & B & - & - & - & - & - & - & - & -2 & ${ }^{1.03}$ \\
\hline Gloeocapsopsis crepidinum (Thuret) Geitler ex Komárek & GloCre & 0 & 0 & 0 & 1 & 0 & 0 & 0 & B & - & - & - & hl & - & - & - & - & e \\
\hline Gloeotrichia pisum Thuret ex Bornet \& Flahault & GlePis & 0 & 0 & 0 & 1 & 0 & 0 & 0 & B,Ep & - & - & ind & $\mathrm{hl}$ & - & $\mathrm{o}$ & - & o-a & 1.8 \\
\hline Johannesbaptiststia pellucida (Dickie) W.R.Taylor \& Drouet & JohPel & ${ }_{0}^{0}$ & 0 & 1 & 1 & 1 & 1 & 1 & B & - & - & - & hlbnt & - & - & - & - & - \\
\hline $\begin{array}{l}\text { Leptolyng byy cebennensis (Gomont) I.Umezaki \& M.Watanabe } \\
\text { Phhormidium }\end{array}$ & $\begin{array}{lll}\text { LepCeb } \\
\text { PhoAtr }\end{array}$ & $0_{0}^{0}$ & 0 & 0 & ${ }_{0}^{1}$ & 0 & 1 & 1 & $\mathrm{Ep}, \mathrm{S}$ & - & aer & - & - & - & o & - & - & - \\
\hline $\begin{array}{l}\text { Phormidium marticulalum m (N.L.GGardner) Anagnostidis \& Komárek } \\
\text { Phormidium chlorinum (Kützing ex Gomont) Umezaki \& Watanabe }\end{array}$ & $\begin{array}{l}\text { PhoArt } \\
\text { Phochl }\end{array}$ & ${ }_{0}^{0}$ & $\begin{array}{l}0 \\
0\end{array}$ & $\begin{array}{l}1 \\
0\end{array}$ & $\begin{array}{l}0 \\
0\end{array}$ & $\begin{array}{l}1 \\
0\end{array}$ & $\begin{array}{l}0 \\
1\end{array}$ & $\begin{array}{l}1 \\
1\end{array}$ & P-B, & & st-str, $\mathrm{H}_{2} \mathrm{~S}$ & - & 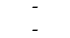 & - & - & - & $\dot{b-p}$ & 3.8 \\
\hline Phormidium kuetzingianum (Kirchner ex Hansgirg) Anagnostidis \& Komárek & PhoKue & 0 & 0 & 1 & 0 & 0 & 0 & 0 & $\mathrm{~B}, \mathrm{~S}$ & - & aer & - & - & - & - & - & Dop & 3.8 \\
\hline Phormidium puteale (Montagne ex Gomont) Anagnostidis \& Komárek & PhoPut & 0 & 0 & 0 & 0 & 0 & 1 & 1 & $\mathrm{~B}, \mathrm{~S}$ & . & st-str & - & - & - & - & - & - & - \\
\hline Pseudophormidium spelaeoides (Cado) Anagnostidis & Psespe & 0 & 1 & 1 & 1 & 0 & 0 & 1 & & - & & - & - & - & - & - & & -5 \\
\hline Rhabdogloea smithii (Chodat \& F.Chodat) Komárek & RhaSmi & 0 & 0 & 0 & 0 & 0 & 0 & 1 & $\mathrm{P}$ & ( & st & - & - & - & $\mathrm{o}$ & - & 0 & 1.1 \\
\hline Synechocystis aquatilis Sauvageau & SynAqu & 1 & 0 & 0 & 0 & 0 & 0 & 0 & P-B & warm & - & - & - & - & $0-m$ & - & b-o & 1.6 \\
\hline Synechocystis pevalekii Ercegovic & SynPev & 1 & 0 & 0 & 0 & 1 & 0 & 0 & $\mathrm{Ep}, \mathrm{S}$ & - & - & & & - & - & & & \\
\hline Synechocystis sallensis Skuja & SynSal & 1 & 0 & 0 & 0 & 0 & 0 & 0 & P-B & - & - & - & - & - & - & - & - & - \\
\hline \multicolumn{19}{|l|}{ Synechocystis sallensis Skuja } \\
\hline Achnanthidium deflexum (C.W.Reimer) J.C.Kingston & AchDef & & & & & & & & B & - & - & - & - & es & - & - & - & 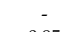 \\
\hline Achnanthidium minutissimum (Kützing) Czarnecki & AchMin & 1 & 1 & 0 & 1 & 1 & 1 & 1 & P-B & eterm & st-str & ind & $\mathrm{i}$ & es & o-e & ate & $x-b$ & 0.95 \\
\hline Adlafia minuscula (Grunow) Lange-Bertalot & AdlMin & 1 & 0 & 0 & 1 & 0 & 0 & 0 & P-B & - & st-str & ind & - & es & ot & - & $a-0$ & 2.8 \\
\hline Adlafai suchlandtii (Hustedt) Monnier \& Ector & AdlSuh & 1 & 0 & 0 & 0 & 0 & 0 & 0 & B & & str & ind & $\mathrm{hb}$ & $s x$ & ot & ats & o & \\
\hline Amphora inariensis Krammer & AmpIna & 1 & 1 & 0 & 0 & 0 & 0 & 0 & B & - & - & alf & oh & - & $o-m$ & - & $0-x$ & 0.7 \\
\hline Cavinula weeinzierlii (H.Schimanski) D.B.Czarnecki & CavWei & 1 & & & 0 & 0 & 0 & 0 & & & 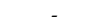 & & & & & & & \\
\hline Cocconeis placentula Ehrenberg & CocPla & 0 & 0 & 0 & 1 & 0 & 0 & 0 & P-B & temp & st-str & alf & $\mathrm{i}$ & es & me & ate & o & 1.35 \\
\hline Craticula halophilioides (Hustedt) Lange-Bertalot & CraHal & 0 & 1 & 0 & 1 & 0 & 0 & 0 & B & & - & & hl & es & & - & & \\
\hline Cyclotella cretica var: cyclopuncta (H. Hakansson \& J.R.Carter) R.Schmidt & CycCre & 0 & 1 & 0 & 0 & 0 & 0 & 1 & & - & - & - & - & - & ot & - & o & 1 \\
\hline nbiformis C.Agardh & CymCym & 0 & 0 & 0 & 1 & 1 & 1 & 1 & B & temp & str & ind & $\mathrm{i}$ & $\mathrm{sx}$ & $o-m$ & ats & b & 2 \\
\hline Cymbella helvetica Kützing & CymHel & 0 & 0 & 1 & 1 & 1 & 1 & 1 & B & & & & $\mathrm{i}$ & (2) & - & (a) & & \\
\hline Cymbella tumida (Brébisson) Van Heurck & CymTum & 0 & 0 & 0 & 1 & 1 & 0 & 0 & B & temp & str & alf & $\mathrm{i}$ & $\mathrm{sx}$ & me & ats & $\mathrm{b}$ & 2.2 \\
\hline $\begin{array}{l}\text { Cymbella a tumididua a runow } \\
\text { Cymbopleura subaequalis (Grunow) Krammer }\end{array}$ & $\begin{array}{l}\text { CymTul } \\
\text { Cymubu }\end{array}$ & $\begin{array}{l}0 \\
1\end{array}$ & $\begin{array}{l}0 \\
0\end{array}$ & $\begin{array}{l}0 \\
0\end{array}$ & $\begin{array}{l}1 \\
0\end{array}$ & $\begin{array}{l}1 \\
1\end{array}$ & $\begin{array}{l}1 \\
0\end{array}$ & $\begin{array}{l}1 \\
1\end{array}$ & в & $:$ & str & neu & $\mathrm{hb}$ & sx & o-m & ats & o & 1 \\
\hline Diploneis oculata (Brébisson) Cleve & DipOcu & 1 & 0 & 0 & 0 & 0 & 0 & 0 & B & - & ( & alf & i & si & - & - & - & \\
\hline Encyonopsis microcephala (Grunow) Krammer & EncMic & 0 & 0 & 0 & 1 & 1 & 1 & 1 & B & - & str & alf & $\mathrm{i}$ & es & me & ats & o & 1.3 \\
\hline Eunotia bilunaris (Ehre & EunBil & 0 & 1 & 0 & 0 & 0 & 0 & 0 & B & temp & st-str & acf & $\mathrm{i}$ & es & o-e & ate & $\mathrm{o}$ & 1 \\
\hline Fal & FalLen & & 0 & 0 & 1 & 0 & 0 & 1 & B & & & & & & & & & 1.1 \\
\hline ickle \& D.G.Mann & FalPyg & 1 & 0 & 0 & 0 & 0 & 0 & 0 & P-B & - & st-str & alf & $\mathrm{mh}$ & es & e & hne & a-o & 2.7 \\
\hline Fallacia vitrea (Østrup) D & FalVit & 0 & 1 & 0 & 0 & 0 & 1 & 1 & B & - & - & & $-\bar{i}$ l & - & - & - & $x-b$ & 0.9 \\
\hline $\begin{array}{l}\text { Fragilaria acus (Kützing) Lange-Bertalot } \\
\text { Fragilaria capucino Desmazieres }\end{array}$ & $\begin{array}{l}\text { FraAcu } \\
\text { Fracap }\end{array}$ & ${ }_{1}^{0}$ & $\frac{1}{0}$ & ${ }_{0}^{0}$ & 1 & 1 & ${ }_{0}^{0}$ & ${ }_{0}^{0}$ & $\begin{array}{c}P \\
P-B\end{array}$ & : & st-str & alb & $\mathrm{i}$ & es & - & - & ${ }^{o-a}$ & 1.8 \\
\hline $\begin{array}{l}\text { Fragilaria capucina Desmazieres } \\
\text { Frustulia rhomboides (Fhrenberg) De Toni }\end{array}$ & $\begin{array}{l}\text { FraCap } \\
\text { FruRho }\end{array}$ & ${ }_{0}^{1}$ & 1 & ${ }_{0}^{0}$ & $\frac{1}{0}$ & $\frac{1}{0}$ & ${ }_{0}^{0}$ & ${ }_{0}^{0}$ & $\begin{array}{c}\text { P-B } \\
\text { B }\end{array}$ & - & st & $\begin{array}{l}\text { ind } \\
\text { acf }\end{array}$ & 1 & 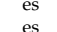 & $\mathrm{m}$ & ats & ${ }^{b-o}$ & 1.6 \\
\hline $\begin{array}{l}\text { Fristulua anhombondes (Enrenberg) De loni } \\
\text { Frustulia vulgaris (Thwaites) De Toni }\end{array}$ & $\begin{array}{l}\text { Frukno } \\
\text { FruVul }\end{array}$ & 0 & 1 & 0 & 0 & 0 & 0 & 0 & $\begin{array}{c}\mathrm{B} \\
\mathrm{P}-\mathrm{B}\end{array}$ & - & $\begin{array}{l}\text { St } \\
\text { st }\end{array}$ & 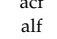 & $\begin{array}{c}\text { no } \\
i\end{array}$ & $\begin{array}{l}\text { es } \\
\text { es }\end{array}$ & $\begin{array}{l}\text { ot } \\
\text { me }\end{array}$ & 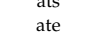 & $\begin{array}{c}x \\
0-a\end{array}$ & 1.8 \\
\hline zing) Rabenhorst & GomAng & 1 & 1 & 1 & 0 & 0 & 0 & 0 & B & - & st-str & ind & $\mathrm{i}$ & es & $0-m$ & (- & o & 1.3 \\
\hline Gomp & GomAug & 1 & 0 & 0 & 1 & 0 & 0 & 1 & B & - & str & ind & $\mathrm{i}$ & es & me & ats & $\mathrm{o}-\mathrm{b}$ & 1.5 \\
\hline Gomphonema grovei var. lingulatum (Hustedt) Lange-Bertalot & GomGro & 1 & 0 & 0 & 0 & 0 & 0 & 0 & B & - & str & & & es & & - & b-a & 2.4 \\
\hline Gomphonema minutum (C.Agardh) C.Agardh & GomMin & 1 & 1 & 0 & 0 & 0 & 0 & 0 & B & - & & alf & $\mathrm{i}$ & es & e & - & o-b & 1.5 \\
\hline Gomphonema olivaceum (Hornemann) Brébisson & Gomoli & 1 & ${ }_{0}^{0}$ & 0 & ${ }_{0}^{0}$ & ${ }_{0}^{0}$ & 0 & 1 & B & - & st-str & alf & $\mathrm{i}$ & & $\mathrm{e}$ & ate & ${ }^{\mathrm{o}-\mathrm{b}}$ & 1.45 \\
\hline $\begin{array}{l}\text { Gomphonema truncatum Enhenberg } \\
\text { Gyrosigma acuminatum (Kützin) Rabenhorst }\end{array}$ & 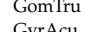 & $\begin{array}{l}1 \\
1\end{array}$ & $\begin{array}{l}0 \\
1\end{array}$ & $\begin{array}{l}0 \\
0\end{array}$ & $\begin{array}{l}0 \\
0\end{array}$ & $\begin{array}{l}0 \\
0\end{array}$ & $\begin{array}{l}0 \\
0\end{array}$ & 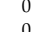 & $\begin{array}{l}\text { B } \\
\text { B }\end{array}$ & - & $\begin{aligned} \text { st-str } \\
\text { st-str }\end{aligned}$ & ind & 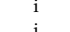 & $\begin{array}{l}\text { es } \\
\text { os }\end{array}$ & me & ats & ${ }^{o-b}$ & 1.4 \\
\hline $\begin{array}{l}\text { Humidophila contenta (Grunow) Lowe, Kociolek, J.R.J.Johansen, Van de Vijver, Lange-Bert } \\
\text { a }\end{array}$ & $\begin{array}{l}\text { Gyracu } \\
\text { HumCon }\end{array}$ & 1 & 0 & 0 & 0 & 0 & 0 & 0 & B, aer & COOI & $\begin{array}{l}\text { St-str } \\
\text { str }\end{array}$ & $\begin{array}{l}\text { aff } \\
\text { ind }\end{array}$ & $\begin{array}{l}1 \\
\mathrm{i}\end{array}$ & $\begin{array}{l}\text { es } \\
\text { es }\end{array}$ & $\begin{array}{c}\text { me } \\
0-m\end{array}$ & $\begin{array}{l}\text { ate } \\
\text { ate }\end{array}$ & $\begin{array}{c}0-a \\
0-x\end{array}$ & $\begin{array}{l}1.95 \\
0.7\end{array}$ \\
\hline
\end{tabular}


Table 3. Cont.

\begin{tabular}{|c|c|c|c|c|c|c|c|c|c|c|c|c|c|c|c|c|c|c|}
\hline Iconella robusta (Ehrenberg) Ruck \& Nakov & IcoRob & 1 & 0 & 0 & 0 & 0 & 0 & 0 & P-B & - & st-str & ind & $\mathrm{I}$ & es & ot & - & $x-0$ & 0.5 \\
\hline Kobayasiella jangii (Meister) Lange-Bertalot & Kobjaa & 0 & 0 & 0 & 0 & 1 & 1 & 1 & B & - & - & - & - & - & - & - & $\mathrm{x}$ & 0.1 \\
\hline Мауатаеа atomus (Kützing) Lange-Bertalot & MayAto & 1 & 1 & 1 & 1 & 0 & 0 & 1 & B & - & st-str & alf & $\mathrm{i}$ & es & he & hce & $a-o$ & 2.6 \\
\hline $\begin{array}{l}\text { Navicula phyllepta Kützing } \\
\text { Nagivecla radioss Kützing }\end{array}$ & NavPhy & 1 & 0 & 0 & 0 & 0 & 0 & 0 & B & - & - & - & $\mathrm{hl}$ & es & - & - & - & - \\
\hline $\begin{array}{l}\text { Navicicla radiosa Kätzing } \\
\text { Navicula veneta Kützing }\end{array}$ & $\begin{array}{l}\text { NavRad } \\
\text { Naylen }\end{array}$ & 1 & 1 & 0 & 0 & ${ }_{0}^{0}$ & ${ }_{0}^{0}$ & 0 & B & temp & st-str & ind & $\mathrm{i}$ & es & me & ate & o & 1.3 \\
\hline $\begin{array}{l}\text { Naviculal veneta Kutzing } \\
\text { Nitzschia amphibioides Hustedt }\end{array}$ & $\begin{array}{l}\text { NavVen } \\
\text { NitAmp }\end{array}$ & 0 & 10 & o & 1 & o & 1 & 1 & $\begin{array}{c}\mathrm{B} \\
\mathrm{P}-\mathrm{B}\end{array}$ & 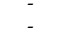 & st-str & $\begin{array}{l}\text { alt } \\
\text { alf }\end{array}$ & $\begin{array}{c}\mathrm{hl} \\
\mathrm{i}\end{array}$ & es & - & - & a-o & 2.7 \\
\hline Nitzschia dissipata (Kützing) Rabenhorst & NitDis & 1 & 1 & 0 & 0 & 0 & 0 & 0 & $\mathrm{~B}$ & - & st-str & alf & $\mathrm{i}$ & sx & me & ate & $\mathrm{b}-\mathrm{o}$ & 1.7 \\
\hline Nitzschia dubiiformis Hustedt & NitDub & 0 & 0 & 1 & 0 & 0 & 0 & 0 & & - & & & $\mathrm{hl}$ & & & - & & \\
\hline Nitzschia gracilis Hantzsch & NitGra & 0 & 0 & 0 & 0 & 0 & 1 & 1 & P-B & temp & st-str & ind & $\mathrm{i}$ & sp & $\mathrm{m}$ & - & o-a & 1.8 \\
\hline Nitzschia intermedia Hantzsch & $\begin{array}{l}\text { Nittnt } \\
\text { Nittan }\end{array}$ & 1 & 0 & 0 & 0 & 0 & 0 & 0 & P-B & - & - & ind & $\mathrm{i}$ & es & e & - & $\mathrm{b}$ & 2 \\
\hline $\begin{array}{l}\text { Nitzschia lanceolata W.Smith } \\
\text { Nitrschin linearic WSmith }\end{array}$ & $\begin{array}{l}\text { NitLan } \\
\text { Nittion }\end{array}$ & 0 & 0 & 0 & 0 & 0 & 1 & 1 & $B$ & $\therefore$ & - & alf & $\mathrm{hl}$ & - & e & - & $\mathrm{b}$ & 2 \\
\hline $\begin{array}{l}\text { Nitzzcchia lineariri W.S.Sinth } \\
\text { Nitschia palae (Kützing) W.Smith }\end{array}$ & $\begin{array}{l}\text { Nittin } \\
\text { Nitery }\end{array}$ & 0 & 0 & 1 & 0 & 0 & 1 & 1 & B & temp & st-str & alf & $\mathrm{i}$ & es & me & ate & b-o & 1.7 \\
\hline $\begin{array}{l}\text { Nitzzchia a alea (Kützing) W.Smith } \\
\text { Nitzschia paleacea (Grunow) Grunow }\end{array}$ & $\begin{array}{l}\text { Nittal } \\
\text { NitPae }\end{array}$ & $\begin{array}{l}1 \\
1\end{array}$ & $\begin{array}{l}0 \\
1\end{array}$ & $\begin{array}{l}0 \\
0\end{array}$ & ${ }_{1}^{0}$ & $\begin{array}{l}0 \\
0\end{array}$ & $\begin{array}{l}0 \\
0\end{array}$ & 0 & $\begin{array}{l}P-B \\
P-B\end{array}$ & temp & - & ind & $\mathrm{i}$ & sp & he & $\begin{array}{l}\text { hee } \\
\text { hee }\end{array}$ & $a-o$ & 2.8 \\
\hline $\begin{array}{l}\text { Nitzschaia palecacea (rrunow) Grunow } \\
\text { Nitzscha pusilla Grunow }\end{array}$ & $\begin{array}{l}\text { Nittrae } \\
\text { NitPus }\end{array}$ & 1 & 0 & o & $l_{0}^{1}$ & o & o & 0 & $\begin{array}{l}P-B \\
P-B, S\end{array}$ & - & 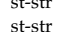 & $\begin{array}{l}\text { alf } \\
\text { alf }\end{array}$ & $\begin{array}{l}1 \\
\mathrm{i}\end{array}$ & 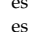 & $\begin{array}{c}\mathrm{e} \\
\mathrm{o-e}\end{array}$ & $\begin{array}{l}\text { hee } \\
\text { tate }\end{array}$ & $\begin{array}{c}b \\
b-0\end{array}$ & 2.2 \\
\hline Nitzschia solita Hustedt & Nitsol & 0 & 0 & 0 & 0 & 0 & 0 & 1 & $B$ & . & st & alf & $\mathrm{mh}$ & es & $\mathrm{e}$ & - & $a-b$ & 3.6 \\
\hline Pinnularia polyonca (Brébisson) W.Smith & PinPol & 0 & 1 & 0 & 0 & 0 & 0 & 0 & B & - & str & acf & $\mathrm{I}$ & - & ot & ats & $x$ & $\begin{array}{l}0.0 \\
0.2\end{array}$ \\
\hline Planothidium lanceolatum (Brébisson ex Kützing) Lange-Bertalot & PlaLan & 0 & 0 & 0 & 0 & 0 & 0 & 1 & P-B & warm & st-str & alf & $\mathrm{i}$ & sx & e & ate & b-o & 1.6 \\
\hline & SelPup & 1 & 0 & 0 & 0 & 0 & 0 & 0 & В & eterm & st & ind & $\mathrm{hl}$ & $\mathrm{sx}$ & me & ate & o-a & 1.9 \\
\hline Staurosira construens var. exigua (W.Smith) H.Kobayasi & & 1 & 0 & 0 & 0 & 0 & 0 & 0 & B & & str & & & sp & me & ate & o & 1.3 \\
\hline Tryblionella scalaris (Ehrenberg) Siver \& P.B.Hamilton & Trysca & 1 & 1 & 1 & 0 & 0 & 0 & 0 & B & - & . & alf & $\mathrm{mh}$ & & $\mathrm{e}$ & & & - \\
\hline Chlorophyta & & & & & & & & & & & & & & & & & & \\
\hline Ankistrodesmus falcatus (Corda) Ralfs & AnkFal & 0 & 1 & 0 & 0 & 0 & 0 & 0 & $\mathrm{P}-\mathrm{B}$ & - & st-str & - & $\mathrm{hb}$ & - & - & - & $\mathrm{b}$ & 2.3 \\
\hline Gloeocystis vesiculosa Nägeli & Gloves & 0 & 1 & 0 & 1 & 0 & 0 & 0 & P-B, S & - & - & - & - & - & - & - & - & - \\
\hline Monoraphidium grififthii (Berkeley) Komárková-Legnerová & MonGri & 0 & 1 & 0 & 0 & 0 & 0 & 0 & P-B & - & st-str & & $\mathrm{i}$ & - & - & - & $\mathrm{b}$ & 2.2 \\
\hline Neglectella solitaria (Wittrock) Stenclová \& Kastovsky & NegSol & 1 & 0 & 0 & 1 & 0 & 1 & 1 & $\mathrm{P}$ & - & st & ind & $\mathrm{i}$ & - & - & - & b-o & 1.7 \\
\hline $\begin{array}{l}\text { Oedoginium sp. } 1 \\
\text { Oedoginium sp. } 2\end{array}$ & $\begin{array}{l}\text { Oedo1 } \\
\text { Oedo }\end{array}$ & 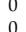 & 0 & 10 & 0 & 1 & 0 & 0 & - & - & - & - & - & - & - & - & - & - \\
\hline $\begin{array}{l}\text { Oedoginiumm sp. } 2 \\
\text { Palmella miniata Leiblein }\end{array}$ & $\begin{array}{l}\text { Oedo2 } \\
\text { PalMin }\end{array}$ & $\begin{array}{l}0 \\
0 \\
0\end{array}$ & $\begin{array}{l}0 \\
0 \\
0\end{array}$ & $\begin{array}{l}0 \\
0 \\
0\end{array}$ & 1 & 0 & 0 & 0 & - & - & - & - & - & $=$ & - & - & - & - \\
\hline Pyrobotryy stellatusu (Korshikov) Korshikov & Pyrste & 0 & 0 & 0 & 1 & 0 & 0 & 0 & - & - & - & - & - & - & - & - & - & - \\
\hline Scenedesmus obtusus Meyen & SceObt & 0 & 0 & 0 & 0 & 0 & 0 & 1 & $P-B$ & - & st-str & & - & - & - & - & o-a & 1.8 \\
\hline Tetradesmus obliquus (Turpin) M.J.Wynne & TetObl & 0 & 0 & 0 & 0 & 0 & 0 & 1 & P-B & - & st-str & ind & $\mathrm{i}$ & - & - & - & $\mathrm{b}$ & 2.05 \\
\hline Trochiscia obtusa (Reinsch) Hansgirg & TroObt & 0 & 1 & 0 & 0 & 0 & 0 & 0 & - & - & - & & - & - & - & - & - & - \\
\hline Charophyta & & & & & & & & & & & & & & & & & & \\
\hline $\begin{array}{l}\text { Cosmarium angulare L.N.Johnson } \\
\text { Spirogrya sp. }\end{array}$ & $\begin{array}{l}\text { CosAng } \\
\text { Spirog }\end{array}$ & $\begin{array}{l}0 \\
0\end{array}$ & $\begin{array}{l}0 \\
0\end{array}$ & $\begin{array}{l}0 \\
0\end{array}$ & $\begin{array}{l}0 \\
0\end{array}$ & $\begin{array}{l}0 \\
1\end{array}$ & $\begin{array}{l}0 \\
0\end{array}$ & $\begin{array}{l}1 \\
0\end{array}$ & $\begin{array}{l}\text { B } \\
-\end{array}$ & - & - & ind & - & - & $\stackrel{\mathrm{e}}{-}$ & - & - & - \\
\hline $\begin{array}{c}\text { Rhodophyta } \\
\text { Porphyridium purpureum (Bory) K.M.Drew \& R.Ross }\end{array}$ & PorPur & 0 & 1 & 1 & 0 & 0 & 0 & 1 & - & - & - & - & - & - & - & - & - & - \\
\hline
\end{tabular}

Note, abbreviations of the site names are as in Tables 1 and 2. Taxa present, 1; taxa absent, 2. Ecological groups substrate: S, soil; Ep, epiphytes; B, benthic as a whole; $\mathrm{P}-\mathrm{B}$, planktonic-benthic; P, planktonic; aer, aerophites. Temperature: cool, cool loving; temp, temperate temperature waters; eterm, eurythermal; warm, warm waters. Water disturbance and Oxygenation: aer, aerophiles; str, streaming well oxygenated waters; st-str, low streaming, middle oxygenated waters; st, standing, low-oxygenated waters; $\mathrm{H}_{2} \mathrm{~S}$, sulfide indicators. Water $\mathrm{pH}$ : acf, acidophiles; ind, $\mathrm{pH}$-indifferents including neutrophiles; alf, alkaliphiles; alb, alkalibiontes. Salinity: hb, halophobes; $\mathrm{i}$, chloride-tolerated (indifferent); hl, halophiles; mh, mesohalobes; hbnt, halobiontes. Saprobity according Watanabe: sx, saproxenes; es, eurysaprobes; sp, saprophiles. Saprobity according Sládeček: $\mathrm{x}$, xenosaprobiont; $\mathrm{x}-\mathrm{o}$, xeno-oligosaprobiont; $\mathrm{x}-\mathrm{b}$, xeno-beta-mesosaprobiont; o, oligosaprobiont; o-b, oligo-beta-mesosaprobiont; b-o, beta-oligosaprobiont; o-a, oligo-alpha-mesosaprobiont; $\mathrm{b}$, beta-mesosaprobiont; $\mathrm{b}-\mathrm{a}$, beta-alpha-mesosaprobiont; $\mathrm{a}-\mathrm{o}$, alpha-oligosaprobiont; $\mathrm{b}-\mathrm{p}$, beta-polysaprobiont; a-b, alpha-beta-mesosaprobiont. Trophy: o, ot, oligotraphentes; o-m, oligo-mesotraphhentes; m, mesotraphentes; me, meso-eutraphentes; e, eutraphentes; o-e, from oligo to eutraphentes; he, hypereutraphentes. Nutrition type: ats, strongly autotropes; ate, autotrophic withstand low nitrogen load; hne, particular heterotrophes (mixotrophes); hce, mixotrophes permanently in need of nitrogen load. 


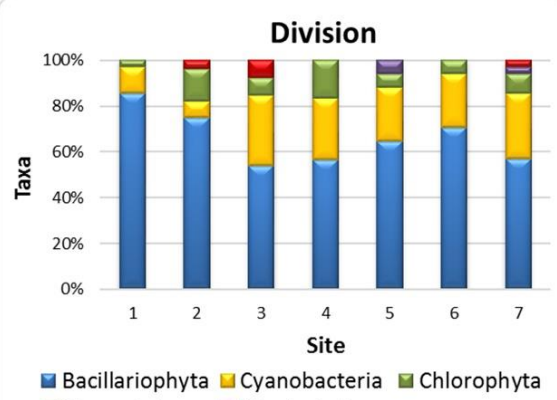

a Charophyta $\square$ Rhodophyta

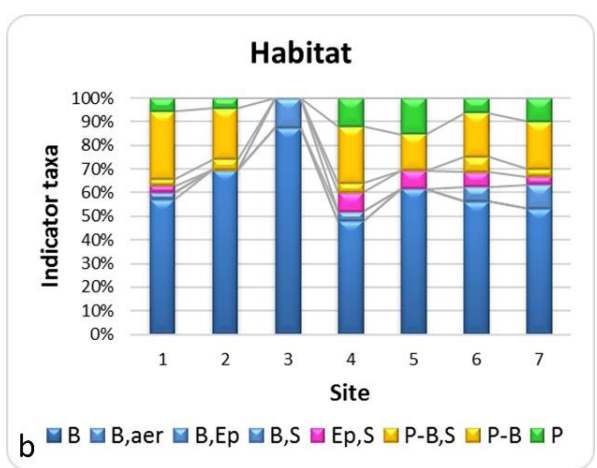

Figure 7. Distribution of the taxonomical content (a) and substrate type indicators (b) in the studied sites of the Abraham Lincoln Birthplace National Historical Park. Abbreviations of site names follow those used in Tables 1 and 2 and abbreviation of ecological groups follow those used in Table 3. Ecological groups of substrate preferences: S, soil; Ep, epiphytes; B, benthic as a whole; P-B, planktonic-benthic; and $\mathrm{P}$, planktonic; aer, aerophites.
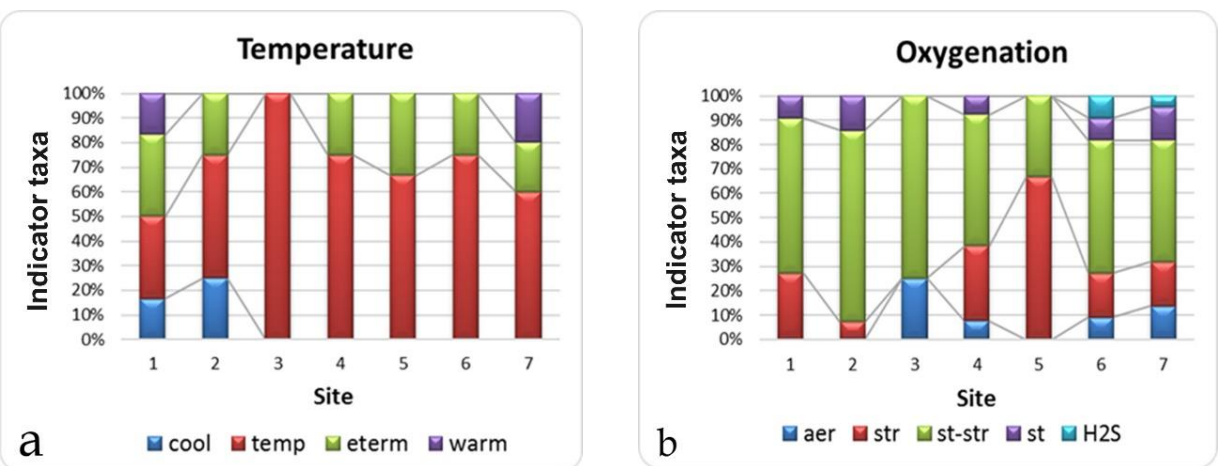

Figure 8. Ecological groups of water temperature preferences (a) and oxygenation (b) indicators in the communities of studied sites in the Abraham Lincoln Birthplace National Historical Park. Abbreviations of site names follow those used in Tables 1 and 2. Abbreviation of ecological groups as in Table 3. Ecological groups of water temperature: cool, cool preferring; temp, temperate temperature waters; eterm, eurythermal; warm, warm waters. Indicators of water disturbance and oxygenation: aer, aerophiles; str, streaming well oxygenated waters; st-str, low streaming, middle oxygenated waters; st, standing, low-oxygenated waters; $\mathrm{H}_{2} \mathrm{~S}$, sulfide indicators.
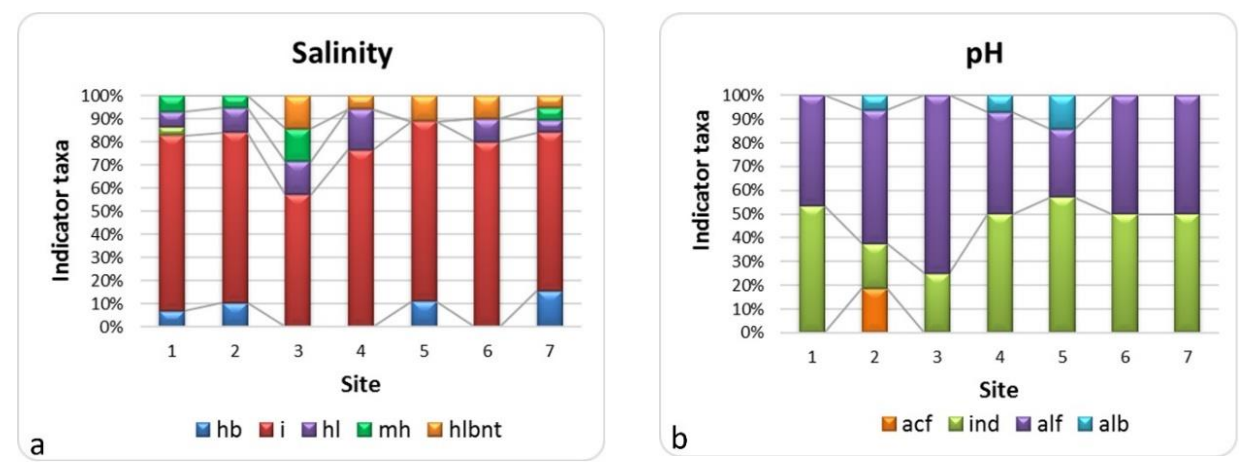

Figure 9. Ecological preferences of water salinity (a) and $\mathrm{pH}(\mathbf{b})$ indicators in the communities of studied sites in the Abraham Lincoln Birthplace National Historical Park. Abbreviations of site names follow those used in Tables 1 and 2. Abbreviation of ecological groups as in Table 3. Ecological groups of salinity indicators: hb, halophobes; i, chloride-tolerated (indifferent); hl, halophiles; mh, mesohalobes; hbnt, halobiontes. Water $\mathrm{pH}$ indicators: acf, acidophiles; ind, $\mathrm{pH}$-indifferents including neutrophiles; alf, alkaliphiles; alb, alkalibiontes. 

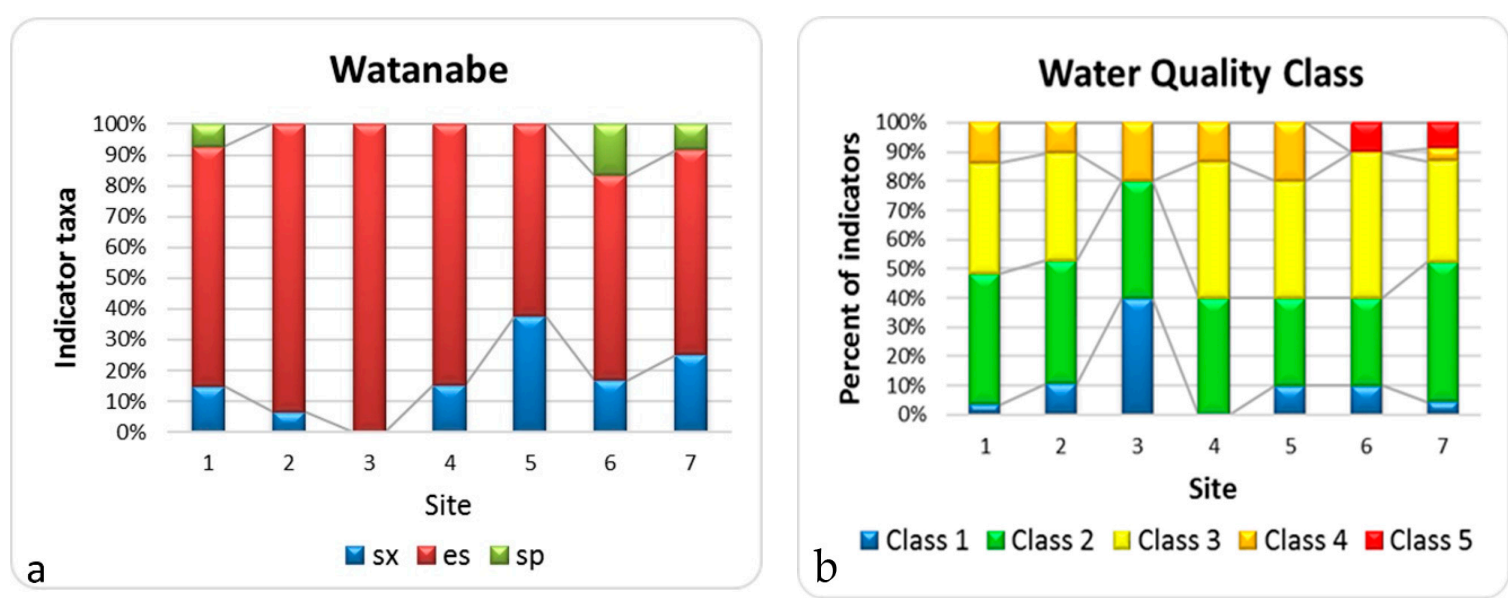

Figure 10. Ecological preferences of organic pollution indicators according to the Watanabe indicator system (a) and water quality classes according to indices of saprobity according to Sládeček (b) in the communities of studied sites in the Abraham Lincoln Birthplace National Historical Park. Abbreviations of site names follow those used in Tables 1 and 2. Abbreviation of ecological groups as in Table 3. Ecological groups of Saprobity indicators according to Watanabe: sx, saproxenes; es, eurysaprobes; sp, saprophiles. Class of water quality indicators are according to Sládeček indices of saprobity: Class 1-5 according to EU color code.
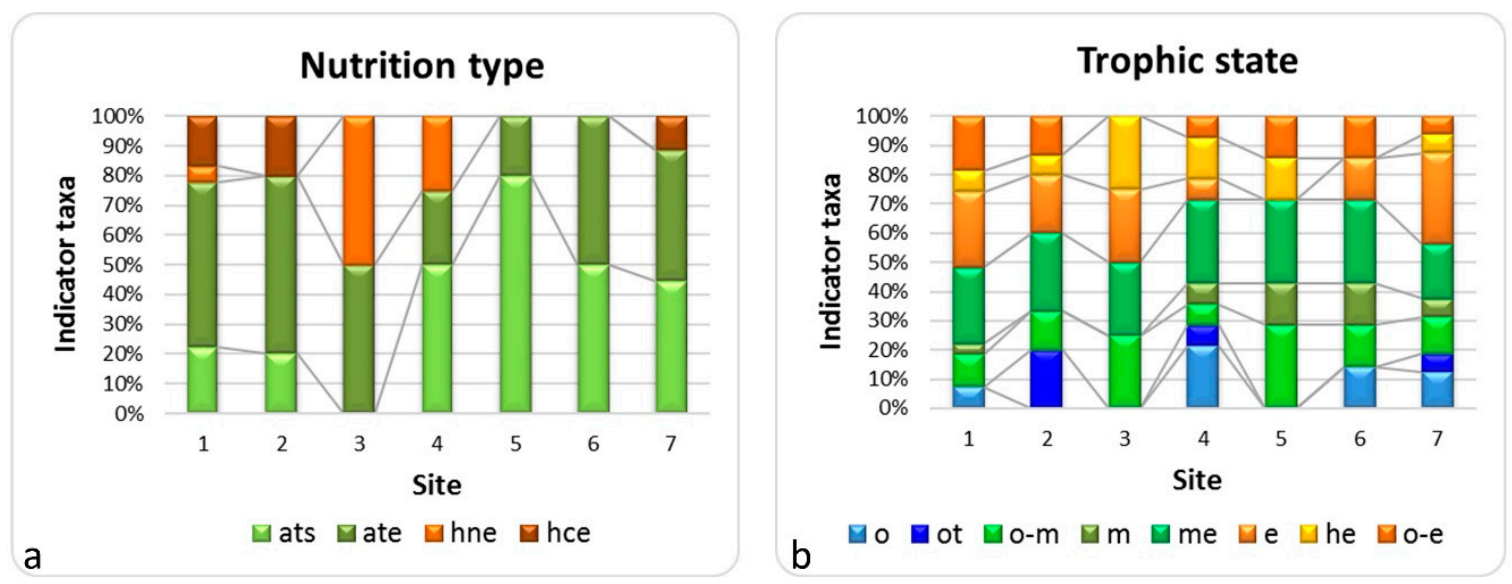

Figure 11. Ecological preferences of algal nutrition type indicators (a) and water trophic states (b) in the communities of studied sites in the Abraham Lincoln Birthplace National Historical Park. Names of sites are abbreviated as in Tables 1 and 2. Abbreviations of ecological groups of nutrition type: ats, strongly autotropes; ate, autotrophic withstand low nitrogen load; hne, particular heterotrophes (mixotrophes); hce, mixotrophes permanently in need of nitrogen load. Indicators of trophic states: $\mathrm{o}$, ot, oligotraphentes; o-m, oligo-mesotraphhentes; m, mesotraphentes; me, meso-eutraphentes; e, eutraphentes; o-e, from oligo- to eutraphentes; he, hypereutraphentes. Autotrophes are green in the histogram and heterotropes are orangish-brown. In the histogram trophic state: oligotraphentes are blue, mesotraphentes are green, and eutraphentes are yellow-orangish. 
Table 4. Calculation of species in taxonomic and bioindicator groups, total species number and index saprobity $\mathrm{S}$ over sites of the Abraham Lincoln Birthplace National Historical Park.

\begin{tabular}{|c|c|c|c|c|c|c|c|}
\hline Group $\backslash$ Site & 1 & 2 & 3 & 4 & 5 & 6 & 7 \\
\hline \multicolumn{8}{|l|}{ Division } \\
\hline Bacillariophyta & 30 & 21 & 7 & 17 & 11 & 12 & 20 \\
\hline Cyanobacteria & 4 & 2 & 4 & 8 & 4 & 4 & 10 \\
\hline Chlorophyta & 1 & 4 & 1 & 5 & 1 & 1 & 3 \\
\hline Charophyta & 0 & 0 & 0 & 0 & 1 & 0 & 1 \\
\hline Rhodophyta & 0 & 1 & 1 & 0 & 0 & 0 & 1 \\
\hline \multicolumn{8}{|l|}{ Habitat } \\
\hline B & 20 & 16 & 7 & 12 & 8 & 9 & 16 \\
\hline B,aer & 1 & 0 & 0 & 0 & 0 & 0 & 0 \\
\hline B,Ep & 0 & 0 & 0 & 1 & 0 & 0 & 0 \\
\hline $\mathrm{B}, \mathrm{S}$ & 0 & 0 & 1 & 0 & 0 & 1 & 3 \\
\hline $\mathrm{Ep}, \mathrm{S}$ & 1 & 0 & 0 & 2 & 1 & 1 & 1 \\
\hline $\mathrm{P}-\mathrm{B}, \mathrm{S}$ & 1 & 1 & 0 & 1 & 0 & 1 & 1 \\
\hline P-B & 10 & 5 & 0 & 6 & 2 & 3 & 6 \\
\hline $\mathrm{P}$ & 2 & 1 & 0 & 3 & 2 & 1 & 3 \\
\hline \multicolumn{8}{|l|}{ Temperature } \\
\hline cool & 1 & 1 & 0 & 0 & 0 & 0 & 0 \\
\hline temp & 2 & 2 & 1 & 3 & 2 & 3 & 3 \\
\hline eterm & 2 & 1 & 0 & 1 & 1 & 1 & 1 \\
\hline warm & 1 & 0 & 0 & 0 & 0 & 0 & 1 \\
\hline \multicolumn{8}{|l|}{ Oxygen } \\
\hline aer & 0 & 0 & 1 & 1 & 0 & 1 & 3 \\
\hline str & 6 & 1 & 0 & 4 & 4 & 2 & 4 \\
\hline st-str & 14 & 11 & 3 & 7 & 2 & 6 & 11 \\
\hline st & 2 & 2 & 0 & 1 & 0 & 1 & 3 \\
\hline $\mathrm{H}_{2} \mathrm{~S}$ & 0 & 0 & 0 & 0 & 0 & 1 & 1 \\
\hline \multicolumn{8}{|l|}{$\mathrm{pH}$} \\
\hline acf & 0 & 3 & 0 & 0 & 0 & 0 & 0 \\
\hline neu & 1 & 0 & 0 & 0 & 1 & 0 & 1 \\
\hline ind & 14 & 3 & 1 & 7 & 3 & 4 & 7 \\
\hline alf & 13 & 9 & 3 & 6 & 2 & 4 & 8 \\
\hline alb & 0 & 1 & 0 & 1 & 1 & 0 & 0 \\
\hline \multicolumn{8}{|l|}{ Salinity } \\
\hline $\mathrm{hb}$ & 2 & 2 & 0 & 0 & 1 & 0 & 3 \\
\hline $\mathrm{i}$ & 21 & 13 & 4 & 13 & 7 & 8 & 13 \\
\hline oh & 1 & 1 & 0 & 0 & 0 & 0 & 0 \\
\hline hl & 2 & 2 & 1 & 3 & 0 & 1 & 1 \\
\hline $\mathrm{mh}$ & 2 & 1 & 1 & 0 & 0 & 0 & 1 \\
\hline hlbnt & 0 & 0 & 1 & 1 & 1 & 1 & 1 \\
\hline \multicolumn{8}{|c|}{ Saprobity Watanabe } \\
\hline sx & 4 & 1 & 0 & 2 & 3 & 1 & 3 \\
\hline es & 21 & 14 & 4 & 11 & 5 & 4 & 8 \\
\hline $\mathrm{sp}$ & 2 & 0 & 0 & 0 & 0 & 1 & 1 \\
\hline \multicolumn{8}{|l|}{ Trophic state } \\
\hline o & 2 & 0 & 0 & 3 & 0 & 1 & 2 \\
\hline ot & 0 & 3 & 0 & 1 & 0 & 0 & 1 \\
\hline $\mathrm{o}-\mathrm{m}$ & 3 & 2 & 1 & 1 & 2 & 1 & 2 \\
\hline $\mathrm{m}$ & 1 & 0 & 0 & 1 & 1 & 1 & 1 \\
\hline me & 7 & 4 & 1 & 4 & 2 & 2 & 3 \\
\hline $\mathrm{e}$ & 7 & 3 & 1 & 1 & 0 & 1 & 5 \\
\hline he & 2 & 1 & 1 & 2 & 1 & 0 & 1 \\
\hline o-e & 5 & 2 & 0 & 1 & 1 & 1 & 1 \\
\hline \multicolumn{8}{|l|}{ Nutrition type } \\
\hline ats & 4 & 2 & 0 & 4 & 4 & 2 & 4 \\
\hline ate & 10 & 6 & 1 & 2 & 1 & 2 & 4 \\
\hline hne & 1 & 0 & 1 & 2 & 0 & 0 & 0 \\
\hline hce & 3 & 2 & 0 & 0 & 0 & 0 & 1 \\
\hline
\end{tabular}


Table 4. Cont.

\begin{tabular}{|c|c|c|c|c|c|c|c|}
\hline Group $\backslash$ Site & 1 & 2 & 3 & 4 & 5 & 6 & 7 \\
\hline \multicolumn{8}{|l|}{ Saprobity Sládeček } \\
\hline $\mathrm{x}$ & 0 & 2 & 0 & 0 & 1 & 1 & 1 \\
\hline$x-0$ & 1 & 0 & 0 & 0 & 0 & 0 & 0 \\
\hline $0-x$ & 2 & 1 & 0 & 0 & 0 & 0 & 0 \\
\hline$x-b$ & 1 & 2 & 0 & 1 & 1 & 2 & 2 \\
\hline o & 5 & 4 & 1 & 3 & 2 & 1 & 4 \\
\hline$o-b$ & 5 & 1 & 0 & 1 & 0 & 0 & 4 \\
\hline b-o & 5 & 1 & 1 & 2 & 1 & 2 & 3 \\
\hline o-a & 3 & 3 & 0 & 2 & 1 & 1 & 2 \\
\hline $\mathrm{b}$ & 2 & 3 & 0 & 3 & 2 & 2 & 3 \\
\hline $\mathrm{b}-\mathrm{a}$ & 1 & 0 & 0 & 0 & 0 & 0 & 0 \\
\hline$a-o$ & 4 & 2 & 1 & 2 & 0 & 0 & 1 \\
\hline b-p & 0 & 0 & 0 & 0 & 0 & 1 & 1 \\
\hline$a-b$ & 0 & 0 & 0 & 0 & 0 & 0 & 1 \\
\hline \multicolumn{8}{|l|}{ Class of Water Quality } \\
\hline Class 1 & 1 & 2 & 2 & 0 & 1 & 1 & 1 \\
\hline Class 2 & 13 & 8 & 2 & 6 & 3 & 3 & 11 \\
\hline Class 3 & 11 & 7 & 0 & 7 & 4 & 5 & 8 \\
\hline Class 4 & 4 & 2 & 1 & 2 & 2 & 0 & 1 \\
\hline Class 5 & 0 & 0 & 0 & 0 & 0 & 1 & 2 \\
\hline Index saprobity $S$ & 1.12 & 1.16 & 1.87 & 1.39 & 1.37 & 1.26 & 1.25 \\
\hline No of Species & 36 & 28 & 13 & 30 & 17 & 17 & 35 \\
\hline
\end{tabular}

Note, numbers of the sites as in Tables 1 and 2 and ecological groups as in Table 2. Class of water quality, class 1-5 according to USA and EU FWD five-colored code.

Salinity indicators in the studied waterbodies of the Abraham Lincoln Birthplace National Historical Park were mostly from indifferent groups accompanied by halophilic species in sites \#1 to \#4 and \#6 and \#7 (Figure 9a). The mesohalobic species also were represented in communities of sites \#1 to $\# 3$ and \#7, but in low numbers. The high salinity indicators, such as halobiontes, were represented in sites \#3 to \#7 by $3 \%$ to $13 \%$. Therefore, the water of the studied sites can be defined as low-to-middle enriched by chlorides.

The water $\mathrm{pH}$ indicator distribution shows a prevalence for indifferents and alkaliphiles in all sites excluding those of site $\# 2$ where acidophilic species are about $18 \%$. Alkalibiontes were found in communities of sites \#2, \#4, and \#5, which contained about $5 \%$ to $15 \%$ of the algae species richness (Figure 9b). Therefore, the waterbodies of the Abraham Lincoln Birthplace National Historical Park can be defined as low-alkaline.

The organic pollution indicators according to the Watanabe system demonstrate low-to-middle organic enrichments of the studied sites (Figure 10a). Saproxenes and eurysaprobes represent about $83 \%$ to $100 \%$ of community but saprophiles, indicators of aquatic organic pollution represented up to $17 \%$ in sites \#1, \#6, and \#7, which helps to define all studied waterbodies in the Abraham Lincoln Birthplace National Historical Park as low organically polluted. The same results can be seen in the indication of organic pollution with indices saprobity S (Figure 10b). Indicator prevalence of class 1 to 3 (up to $90 \%$ ) demonstrated low organically polluted waters in all of the studied sites.

Activity of photosynthetic processes can be revealed with algae nutrition type indicators. Figure 11a shows a strong prevalence of autotrophic species (ats and ate), up to $80 \%$ to $100 \%$ in the studied site communities, excluding site \#3 where the mixotrophes were $50 \%$. Indicators of the trophic state demonstrated a presence of diverse groups of algae, from oligotrophic to hypereutrophic states (Figure 11b). In these groups, the oligotrophic and mesotrophic groups (blue and green in Figure 6b) were represented, up to $50 \%$ to $70 \%$ in different site communities that can be defined in the studied waterbodies of the Abraham Lincoln Birthplace National Historical Park as mostly mesotrophic. The eutrophic indicators were enriched communities in sites \#1 to \#3 and \#7 that can infer some eutrophication trends.

We used a comparative floristic program, GRAPHS, to compare the diversity of the algal communities on both, the division and species level. Figure 12a demonstrates the dividing of division 
species richness into two different clusters based on a similarity level of $70 \%$. The diatoms are represented in one cluster, whereas all other divisions are clustered into two clusters. A comparison of species richness on the sites is shown in the dendrogram of Figure 12b. Two clusters are based on $50 \%$ similarity. The first includes communities from sites \#1, \#2, \#4, and \#7, which are enriched by diatoms in the range of 30 to 36 species (Tables 3 and 4). The other three sites (\#3, \#5, and \#6) have a two-time lower diversity (13-17 taxa) as compared with the other cluster.
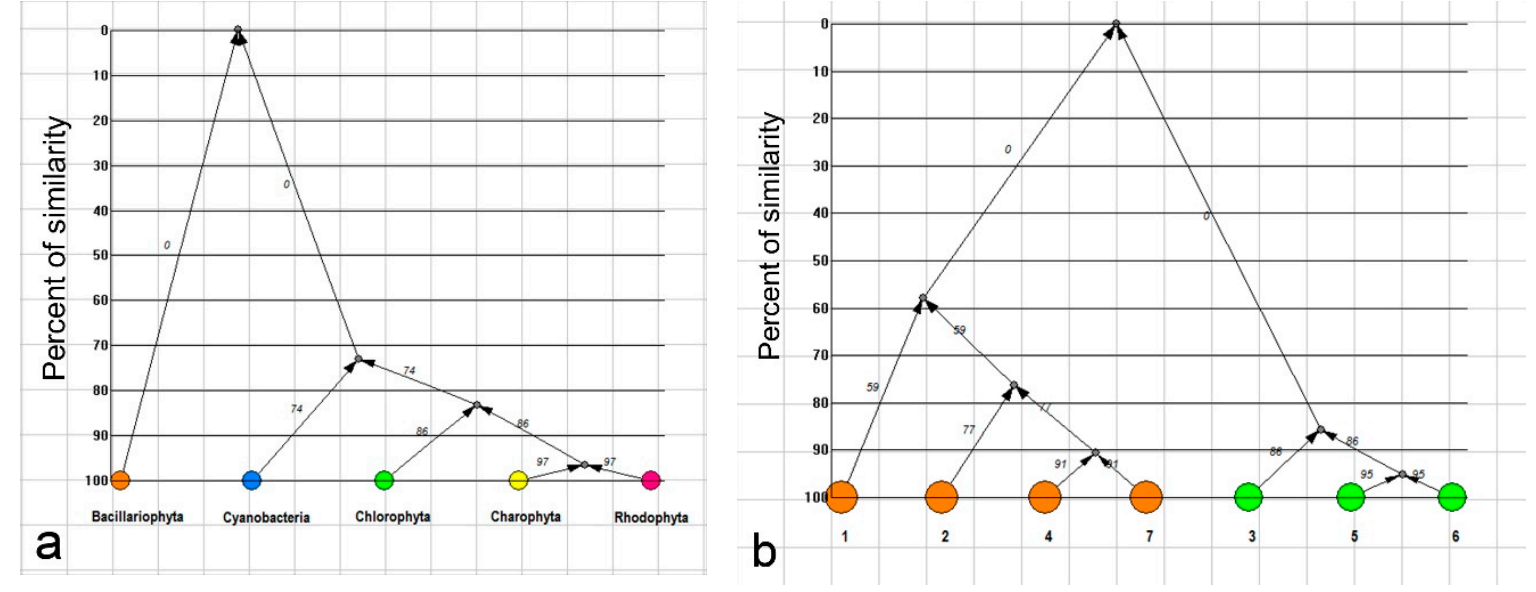

Figure 12. Comparative floristic trees for algal communities in seven studied sites of the Abraham Lincoln Birthplace National Historical Park: taxonomic richness in algal divisions (a), and community species richness $(\mathbf{b})$. The abbreviations for the sites are the same as in Tables 1 and 2.

The statistical analyses were completed to provide clarify of the differences between the floras of all the sites and their relationships with environmental variables. Therefore, the canonical correspondence plot in Figure 10a shows that the environmental variables (Table 2) were divided into three different groups with respect to their influence on algal communities at the division level. Cluster 1 combines sites $\# 1$ and \#2 that are influenced by $\mathrm{Hg}$ and prefer lower water temperatures and $\mathrm{pH}$. Cluster 2 included floras from sites \#4 to \#7 which were influenced by water temperature and $\mathrm{pH}$. Site \#3 (cluster 3) did not show any preferences to environmental factors. We compared the biplot CCA in Figure 13a and triplot with the most abundant species in Figure 13b. This caused the community diversity from the sites to be divided into four clusters in relation to environmental factors. Therefore, algae in sites \#4, \#5, \#7 preferred high $\mathrm{pH}$ (cluster 1) from which Cymbella cymbiformis was a marker taxon. Cluster two included communities at sites \#3 and \#6, which preferred high water temperature with marker species of Achnanthidium deflexum and A. minutissimum. Cluster 3 represented species from site \#1, which preferred nutrients enrichment. Only site \#2 community with Amphora inariensis and Cavinula weinzierlii formed cluster 4 with low dependence with $\mathrm{Hg}$ concentration when TDS was also low.

We compared the species content in the sites' flora with the help of a correlation coefficient calculation. The matrix in Table 5 demonstrates that algal communities in the studied sites of \#1 and $\# 2$, \#2 and \#4, \#2 and \#7, and \#4,\#5,\#6, \#7 were most similar. The JASP correlation plot with the aid of $\mathrm{R}$ statistics was used to combine species diversity to the two most correlated communities with respect to enrichments by diatoms and green algae (sites \#1 and \#2) and diatoms and cyanobacteria (sites \#6 and \#7) (Figure 14a) on the level of similarity $90 \%$. When the similarity filter is about 50\% (Figure 14b), there are four different clusters. The most similar were algae from sites \#6 and \#7, then, sites \#4 and \#5, then, sites \#1 and \#2, and only site \#3 represented the most different community. 

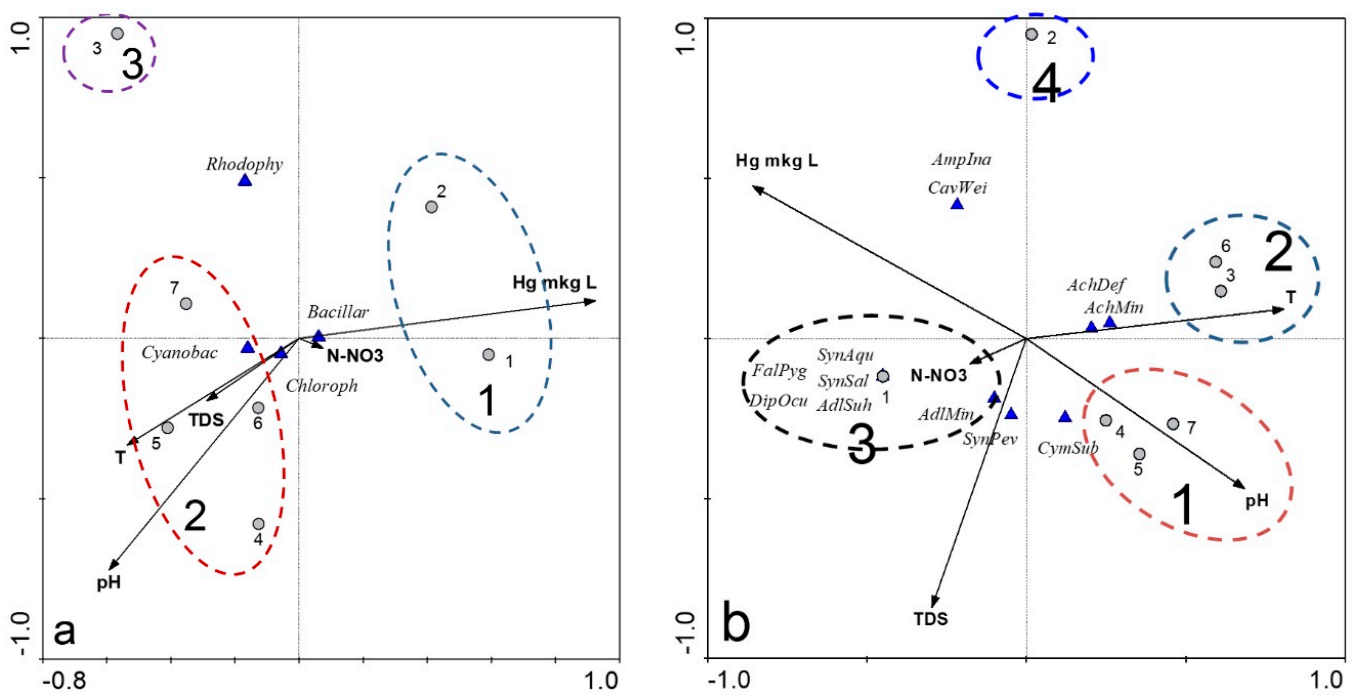

Figure 13. Canonical correspondence plots for algal communities in seven studied site of the Abraham Lincoln Birthplace National Historical Park at the division level and the environmental variables (a), and most abundant species, and environmental variables (b).

Table 5. Similarity matrix for species richness of algae in the Abraham Lincoln Birthplace National Historical Park waterbodies. The abbreviations for the sites are the same as in Tables 1 and 2. The most similar algal communities with coefficients larger than $50 \%$ of similarity are marked in bold.

\begin{tabular}{cccccccc}
\hline Site & $\mathbf{1}$ & $\mathbf{2}$ & $\mathbf{3}$ & $\mathbf{4}$ & $\mathbf{5}$ & $\mathbf{6}$ & $\mathbf{7}$ \\
\hline 1 & $*$ & $\mathbf{5 7 . 8 9}$ & 25.88 & 44.64 & 34.41 & 31.91 & 45.00 \\
2 & $*$ & $*$ & 37.68 & $\mathbf{5 4 . 1 7}$ & 36.36 & 38.46 & $\mathbf{5 1 . 9 2}$ \\
3 & $*$ & $*$ & $*$ & 35.82 & 50.00 & 44.90 & 40.00 \\
4 & $*$ & $*$ & $*$ & $*$ & $\mathbf{5 8 . 6 7}$ & $\mathbf{5 7 . 8 9}$ & $\mathbf{6 0 . 7 8}$ \\
5 & $*$ & $*$ & $*$ & $*$ & $*$ & $\mathbf{6 6 . 6 7}$ & $\mathbf{5 0 . 6 0}$ \\
6 & $*$ & $*$ & $*$ & $*$ & $*$ & $*$ & $\mathbf{6 9 . 0 5}$ \\
7 & $*$ & $*$ & $*$ & $*$ & $*$ & $*$ & $*$
\end{tabular}

Note: ${ }^{*}$ is marked community of site 1 with $100 \%$ of similarity and other that repeated in the right upper part of the Table.
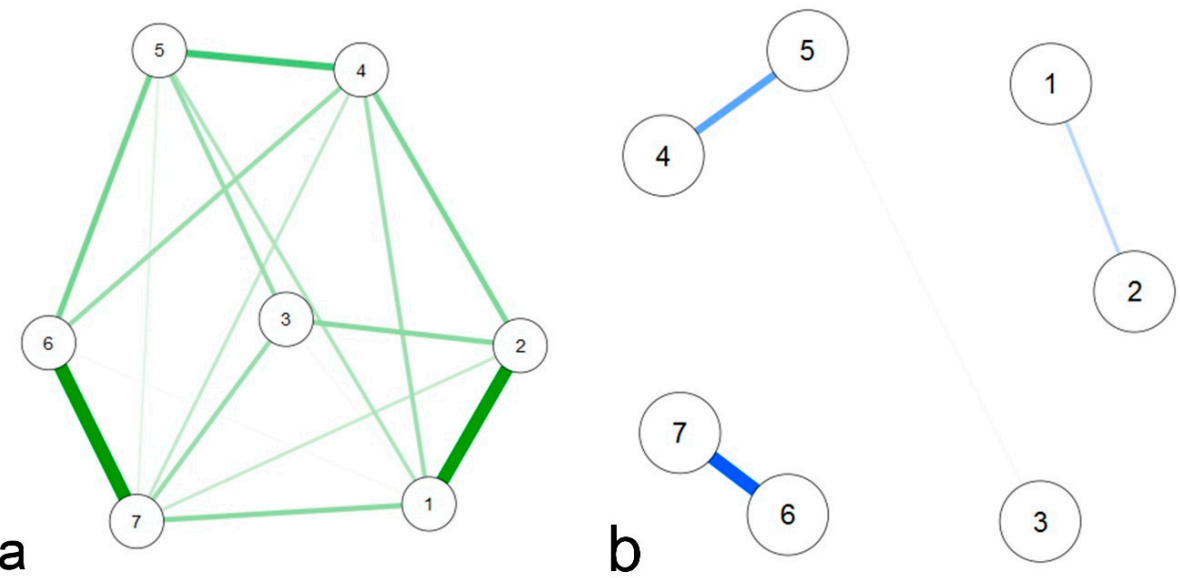

Figure 14. The JASP network graph of correlation of the species richness in seven sites of the Abraham Lincoln Birthplace National Historical Park in similarity level of 50\% (a), and $90 \%$ (b). The abbreviations for the sites are the same as in Tables 1 and 2. Line thickness corresponds to the value of the correlation coefficient. 
The calculation of the percent of unique species in the BioDiversity Pro program (Table 6) showed that site \#1 community included up to $61 \%$ of unique taxa, most of which were diatoms, then followed by sites \#2 (46\%), \#3 (33\%), and \#4 (32\%). The communities of sites \#6 and \#7 were represented by the most common taxa with the lowest uniqueness. It was assumed that diatom diversity plays a major role in the formation of the algal community's process in the Abraham Lincoln Birthplace National Historical Park.

Table 6. Estimator of unique species for communities of algae in the Abraham Lincoln Birthplace National Historical Park waterbodies. The abbreviations for the sites are the same as in Tables 1 and 2.

\begin{tabular}{cccccccc}
\hline Site & $\mathbf{1}$ & $\mathbf{2}$ & $\mathbf{3}$ & $\mathbf{4}$ & $\mathbf{5}$ & $\mathbf{6}$ & $\mathbf{7}$ \\
\hline $\begin{array}{c}\text { Percent of } \\
\text { unique } \\
\text { species }\end{array}$ & 61.2 & 46.8 & 33.6 & 32 & 17.4 & 6.6 & 0 \\
\hline
\end{tabular}

Index saprobity $\mathrm{S}$ was calculated on the bases of species-specific index s from Table 3 and saprobity indicators content on the studied site communities. Table 7 shows that index saprobity had a low range of values and represented class 2 water quality on all sites excluding site \#3. The classification of water quality parameters [47] on the bases of nitric nitrogen concentration (Table 2) and the index saprobity S (Table 4) reflect Class $2-3$ of water quality and available organic enrichments at sites \#1, \#2 and \#6,\#7. We conducted the calculation for the water ecosystem state index (WESI) $[44,48]$ to define the environmental impact on the algal communities from the Abraham Lincoln Birthplace National Historical Park studied sites. Therefore, Table 7 demonstrates that there is little impact on site \#3, which was the only site with a WESI value of more than one, whereas all other site communities were affected and had a WESI index below one.

Table 7. Classification ranks of nitrate-nitrogen concentration and index saprobity S over sites of the Abraham Lincoln Birthplace National Historical Park for calculation of the water ecosystem state index (WESI). The abbreviations for the sites are the same as in Tables 1 and 2. Good ecosystem condition is marked by bold black font. Affected ecosystem condition is marked by bold red font.

\begin{tabular}{|c|c|c|c|c|c|}
\hline Site & $\mathrm{N}-\mathrm{NO}_{3}, \mathrm{mg} \mathrm{L}^{-1}$ & $\mathrm{~N}-\mathrm{NO}_{3}$ Rank & Index S & Index S rank & Index WESI \\
\hline 1 & 2.60 & 8 & 1.12 & 3 & 0.38 \\
\hline 2 & 2.42 & 8 & 1.16 & 3 & 0.38 \\
\hline 3 & 0.44 & 3 & 1.87 & 4 & 1.33 \\
\hline 4 & 1.69 & 6 & 1.39 & 3 & 0.50 \\
\hline 5 & 1.87 & 6 & 1.37 & 3 & 0.50 \\
\hline 6 & 2.10 & 7 & 1.26 & 3 & 0.43 \\
\hline 7 & 4.11 & 9 & 1.25 & 3 & 0.33 \\
\hline
\end{tabular}

\section{Discussion}

Since there has not been previous algal floras studies from the ALBNHP waterbodies, in the first investigative stage, we found 88 taxa of algae and cyanobacteia in seven studied sites from this historical park's waterbodies. This study describes algal community geographical differences. It is not surprising that diatoms predominated, as in some other investigated places in Kentucky, where their species richness has reached from 25 [8] to 279 [13] taxa.

Algae indicators are used in the National Aquatic Resource Surveys $[49,50]$ as part of the rivers and stream water quality assessment program on the bases of density and abundance values for both soft-bodied algae (non-diatom) and diatoms [20]. The diatom bioassessment index (DBI) is a multimetric index, developed in Kentucky based on a calculation of Bacillariophyta algae only (diatom richness, Shannon index, PTI, siltation index, Fragilaria richness, and Cymbella richness) and cannot help in the assessment of contaminated waters [20,51]. However, in many cases, data from diatoms only are not enough for assessing aquatic habitat conditions because they can have a mostly non-diatom 
algal community, however, algae species' autecology is likely correlated with nutrient concentrations. Therefore, bioindicator methods, which included a full species list of community in assessment, can help in the multiparametric indication of aquatic habitats.

From the 17 other studies from Kentucky, the vast majority of the algal species are equally divided between diatoms and green algae with both representing $37.7 \%$ of the community. Cyanobacteria is represented at $15.4 \%$, Dinoflagellates and Euglenoids are both at 3\%, Chrysophytes at $1 \%$, and red algae and Xanthrophytes less than 1\% [2-19,52,53]. In this case, we attribute the diversity of algae that we studied in the Abraham Lincoln Birthplace National Historical Park to a separate type of flora with diatoms in the first place, which is not typical for the flat landscape of Kentucky in a warmer climate.

In comparison to our investigation with other algal floras in Kentucky it can be seen (Figure 15) that the Abraham Lincoln Birthplace National Historical Park algal flora is very different from others previously studied.

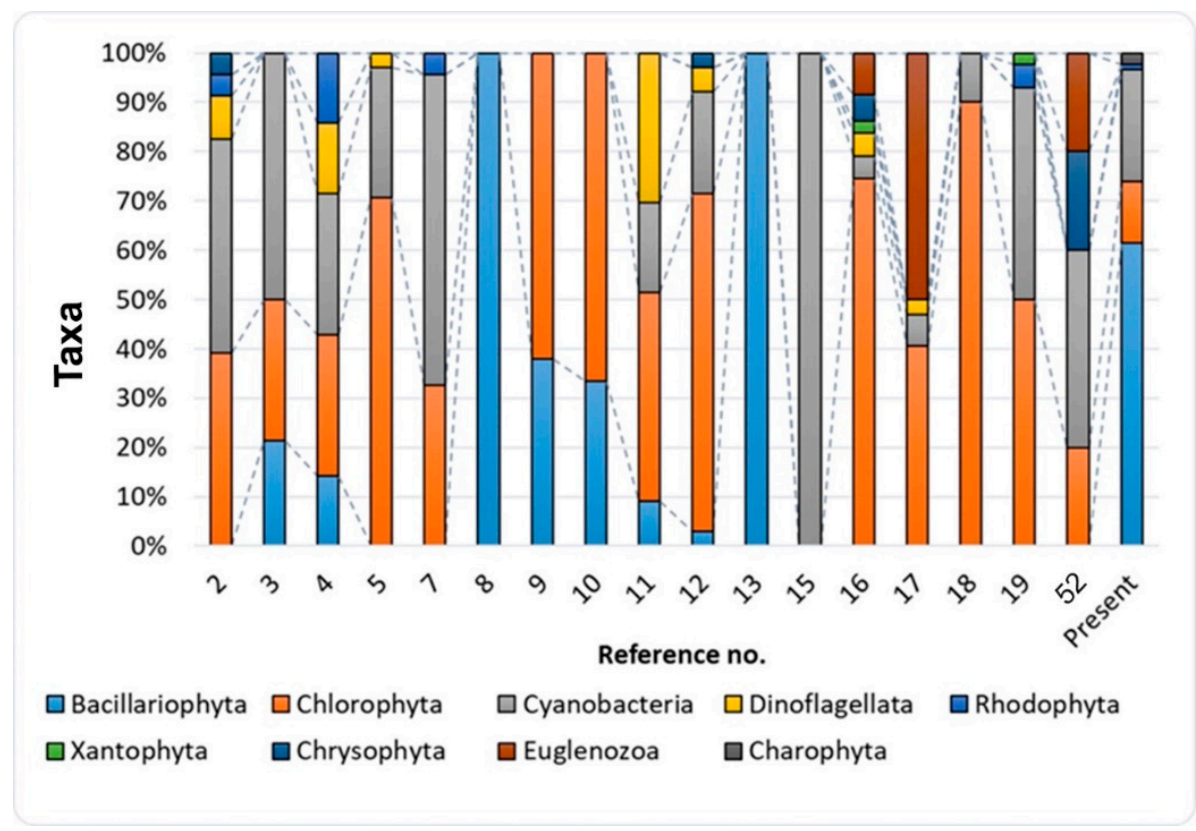

Figure 15. The percentage of the algae species richness in the Abraham Lincoln Birthplace National Historical Park in comparison to the other studied algal floras of Kentucky (Present). The numbers of the references are given on the horizontal axis.

When we glance at the lake flora in Eurasia, it can be seen that diatoms prevailed according to the specific saturation of taxonomic divisions $[45,54,55]$. Therefore, the prevalence of diatom species in the algal floras of the Abraham Lincoln Birthplace National Historical Park can be assessed as peculiarities of this protected area, which can be related to the high heterogeneity of the occupied habitats and the long vegetation period with rather high temperature water, similar to the majority of Eurasian floras $[48,56]$.

Nevertheless, the algal floras in USA have a long history of investigation and bioindicator methods have never been implemented before for the assessment of water quality in contrast to numerous studies in the Old World [46]. Results of our analysis of the ecological preferences of algae and cyanobacteria in the diverse habitat of the Abraham Lincoln Birthplace National Historical Park allows us to characterize the water bodies studied as temperate temperature, well oxygenated in sites \#1 to \#5, but enriched by sulfides in sites \#6 and \#7. The waters are low-alkaline, with low-to-middle salinity, low-to-middle organically polluted, class 1 to 3 water quality, mostly mesotrophic, inhabited by strongly autotrophic species, which formed biomass in plankton, and on submerged substrates. The results are similar to the same assessment of waters in protected areas in Eurasia $[48,56]$. 
This comparative floristic study revealed differences between communities with diatom preference that formed clusters in each type of calculation. The algal communities were divided into groups related to species richness and enriched by diatoms. The CCA analysis of species-environment relationships divided the studied sites, with respect to high $\mathrm{pH}$ and high temperature water species' preferences, from which the markers were identified. The marker species in these studied communities are different from those identified in Eurasian waterbodies $[44,45,48]$ and can be used in the monitoring of the ALBNHP aquatic habitats. Nevertheless, the study sites are under natural protection and influence. We found that site \#1 was organically polluted more than the other six sites. This waterbody is located at a different park location, which the public has easy access to. This site is also groundwater fed which likely comes from outside the park. In order to assess the intensity of anthropogenic impact on the studied aquatic communities, we calculated the index saprobity $S$ and found that water quality can be classified as good natural waters of class 1-3. But when these results were compared to the nitric-nitrogen saturation in each site using the WESI index calculation, we found that site \#3 was the only site that was least impacted, whereas all other site communities were affected, presumably as a result of a small anthropogenic pressure with an influx of nutrients, but not toxicants. This is similar to the estimates in the basin of the agriculturally loaded Songhua River, China [57].

\section{Conclusions}

Algae and cyanobacteria from seven sites were studied for the first time in the Abraham Lincoln Birthplace National Historical Park. There were 88 species identified from which diatoms were dominant. The taxa are divided into four different taxonomic divisions, including a few rare species of red and charophyte algae, but this algal study is centered on the diatom community composition from which one can infer acidification and water temperature as a result of statistical assessments.

Nevertheless, an indication of eutrophication and algae productivity with chlorophyll has been documented in USA [58]. The bioindicator methods of water quality assessment with the use of algae and cyanobacteria species' autoecology were first used in the USA on aquatic habitats during this study. The bioindicator results demonstrated the algal preferences of benthic and planktonic-benthic life styles in temperate temperature middle-oxygenated waters, low-to-middle enriched by chlorides, mostly well oxygenated, but sometimes saturated by sulfides, low-alkaline, low-to-middle organic enriched, class 1 to 3 water quality, and mostly mesotrophic. The majority of observed algal and cyanobacteria diversity of the park was represented by autotrophic species. This reflects the community high self-purification capacity, which is very important for the protection regime control but could not be revealed with chemical analysis only.

Both the bioindicator and statistical methods demonstrated the importance of biological monitoring of waterbodies in the Abraham Lincoln Birthplace National Historical Park in the face of identified anthropogenic pollution in the initial stage on site A.

Therefore, we conclude that the diversity of diatom algae not only plays a major role in the formation of the algal communities and their uniqueness in the studied protected area but diatom algae can also be a good indicator of environmental assessments and change.

Author Contributions: Conceptualization, S.B. and T.S.; data curation, S.B. and T.S.; formal analysis, S.B and T.S.; investigation, T.S.; methodology, S.B.; project administration, S.B.; resources, T.S.; software, S.B.; validation, S.B. and T.S.; visualization, S.B.; writing-original draft, S.B. and T.S.; writing-review and editing, S.B. and T.S.

Funding: This research received no external funding.

Acknowledgments: This work has been partly supported by the Israeli Ministry of Aliyah and Integration.

Conflicts of Interest: The authors declare no conflict of interest. 


\section{References}

1. Muller, R.; McComb, W. Upland Forests of the Knobs Region of Kentucky. Bull. Torrey Bot. Club 1986, 113, $268-280$. [CrossRef]

2. Jones, H. Algological investigations in Mammoth cave, Kentucky. Int. J. Speleol. 1964, 1, 491-516. [CrossRef]

3. Smith, T.; Olson, R. A Taxonomic Survey of Lamp Flora (Algae and Cyanobacteria) in Electrically Lit Passages within Mammoth Cave National Park, Kentucky. Int. J. Speleol. 2007, 36, 105-114. [CrossRef]

4. Nagy, J. Preliminary note on the Algae of Crystal Cave, Kentucky. Int. J. Speleol. 1965, 1, 479-490. [CrossRef]

5. Camburn, K. Subaerial Algae from Eastern Kentucky. Castanea 1983, 48, 83-88.

6. Collier, C. Influence of strip mining on the hydrologic environment of parts of Beaver Creek basin, Kentucky, 1955-1959. U.S. Geol. Surv. Prof. Pap. 1964, 427, 85.

7. Hendricks, S.; Luttenton, M. Benthic Algae Taxa (Exclusive of Diatoms) of the Little River Basin, Western Kentucky, 2000-2003. J. Ky. Acad. Sci. 2007, 68, 31-38. [CrossRef]

8. Hunt, C.; Hendricks, S. Diatom Species Composition and Environmental Conditions at Four Perennial Springs in Western Kentucky and Tennessee. J. Ky. Acad. Sci. 2008, 69, 141-151. [CrossRef]

9. Taylor, W.; Hiatt, F.; Hern, S.; Hilgert, J.; Lambou, V.; Morris, F.; Morris, M.; Thomas, R.; Williams, L. Distribution of Phytoplankton in Kentucky Lakes; EPA-600/3-78-013; U.S. Environmental Protection Agency: Washington, DC, USA, 1978.

10. Yates, J. Influences of a Cladophora Bloom on the Diets of Amblema Plicata and Elliptio Dilatata in the Upper Green River, Kentucky. Master's Thesis, Western Kentucky University, Bowling Green, KY, USA, 2012.

11. Cole, G. Studies on a Kentucky Knobs Lake, III. Some qualitative aspects of the net plankton. Trans. Ky. Acad. Sci. 1959, 18, 88-101.

12. Jarrett, G.; King, J. The Diatom Flora (Bacillariophyceae) of Lake Barkley; DACW62-84-C-85; US Army Corps of Engineers: Nashville, TN, USA, 1989.

13. Jarrett, G.; King, J. Phytoplankton Species in Lake Barkley. Castanea 1991, 56, 90-98.

14. Tehrani, K. The Effects of Phosphorus Enrichment on the Dominant Phytoplankton Communities of Chaney Lake. Master's Thesis, Western Kentucky University, Bowling Green, KY, USA, 1999.

15. Daily, W. Additions to the filamentous Myxomyceae of Indiana, Kentucky and Ohio. Butl. Univ. Bot. Stud. 1945, 7, 132-139.

16. Dillard, G.; Moore, S.; Garrett, L. Kentucky algae, II. Trans. Kentucky Acad. Sci. 1976, 37, $20-26$.

17. Dillard, G.; Crider, S. Kentucky algae, I. Trans. Ky. Acad. Sci. 1976, 31, 66-72.

18. McInteer, B. Distribution of the algae of Kentucky in relation to soil regions. Castanea 1939, 3, 32-35.

19. McInteer, B. Algae of Kentucky: Additions to the Check List of March, 1939. Castanea 1941, 6, 6-8.

20. Flotemersch, J.E.; Stribling, J.B.; Paul, M.J. Concepts and Approaches for the Bioassessment of Non-Wadeable Streams and Rivers; EPA 600-R-06-127; U.S. Environmental Protection Agency: Cincinnati, OH, USA, 2006.

21. Swift, E. Cleaning diatom frustules with ultraviolet radiation and peroxide. Phycologia 1967, 6, 161-163. [CrossRef]

22. Desikachary, T. Cyanophyta; Pyarelal Sah at the Times of India Press: Bombay, India, 1959; p. 68.

23. Dillard, G. Freshwater Algae of the Southeastern United States: Part 1: Chlorophyceae: Volvocales, Tetrasporales and Chlorococcales; Bibliotheca Phycologica Band 81; J. Cramer: Berlin, Stuttgart, Germany, 1989.

24. Dillard, G. Freshwater Algae of the Southeastern United States: Part 3: Chlorophyceae: Zygnematales: Zygnemataceae, Mesotaeniaceae and Desmidiaceae (Section 1); Bibliotheca Phycologica Band 85; J. Cramer: Berlin, Stuttgart, Germany, 1990.

25. Dillard, G. Freshwater Algae of the Southeastern United States: Part 5: Chlorophyceae: Zygnematales: Desmidiaceae (section 3); Bibliotheca Phycologica Band 90; J. Cramer: Berlin, Stuttgart, Germany, 1991.

26. Dillard, G. Freshwater Algae of the southeastern United States: Part 4: Chlorophyceae: Zygnematales: Desmidiaceae (section 2); Bibliotheca Phycologica Band 89; J. Cramer: Berlin, Stuttgart, Germany, 1991.

27. Dillard, G. Freshwater Algae of the Southeastern United States: Part 6: Chlorophyceae: Zygnematales: Desmidiaceae (section 4); Bibliotheca Phycologica Band 93; J. Cramer: Berlin, Stuttgart, Germany, 1993.

28. Dillard, G. Freshwater Algae of the Southeastern United States: Part 2: Chlorophyceae: Ulotrichales, Microsporales, Cylindrocapsales, Sphaeropleales, Chaetophorales, Cladophorales, Schizogoniales, Siphonales and Oedogoniales; Bibliotheca Phycologica Band 83; J. Cramer: Berlin, Stuttgart, Germany, 1989.

29. Forest, H. Handbook of Algae; University of Tennessee Press: Knoxville, TN, USA, 1954; p. 467. 
30. Komárek, J.; Anagnostidis, K. Cyanoprokaryota 1. Teil: Chroococcales. In Süsswasserflora von Mitteleuropa; Ettl, H., Gärtner, G., Heynig, H., Mollenhauer, E., Eds.; Band 19/1; Gustav Fisher: Jena, Germany, 1999; p. 548.

31. Komárek, J.; Anagnostidis, K. Cyanoprokaryota 2. Teil: Oscillatoriales. In Süsswasserflora von Mitteleuropa; Büdel, B., Krienitz, L., Gärtner, G., Schagerl, M., Eds.; Band 19/2; Spektrum Akademischer Verlag, Elsevier GmbH: München, Germany, 2005; p. 759.

32. Krammer, K.; Lange-Bertalot, H. Bacillariophyceae 1. Teil: Naviculaceae. In Süsswasserflora von Mitteleuropa; Ettl, H., Gerloff, J., Heynig, H., Mollenhauer, D., Eds.; Band 2/1; Gustav Fisher: Jena, Germany, 1986; p. 876.

33. Krammer, K.; Lange-Bertalot, H. Bacillariophyceae 2. Teil: Bacillariaceae, Epithemiaceae, Surirellaceae. In Süsswasserflora von Mitteleuropa; Ettl, H., Gerloff, J., Heynig, H., Mollenhauer, D., Eds.; Band 2/2; Gustav Fisher: Jena, Germany, 1988; p. 596.

34. Krammer, K.; Lange-Bertalot, H. Bacillariophyceae 3. Teil: Centrales, Fragilariaceae, Eunotiaceae. In Süsswasserflora von Mitteleuropa; Ettl, H., Gerloff, J., Heynig, H., Mollenhauer, D., Eds.; Band 2/3; Gustav Fisher: Jena, Germany, 1991; p. 598.

35. Krammer, K.; Lange-Bertalot, H. Bacillariophyceae 4. Teil: Achnantaceae. Kritische Ergänzungen zu Navicula (Lineolatae) und Gomphonema. In Süsswasserflora von Mitteleuropa; Ettl, H., Gerloff, J., Heynig, H., Mollenhauer, D., Eds.; Band 2/4; Gustav Fisher: Jena, Germany, 1991; p. 468.

36. Krammer, K.; Lange-Bertalot, H. Bacillariophyceae 5. English and French translation of the keys. In Süsswasserflora von Mitteleuropa; Büdel, B., Gärtner, G., Krienitz, L., Lokhorst, G., Eds.; Band 2/5; Spektrum Akademischer Verlag: Heidelberg-Berlin, Germany, 2000; p. 311.

37. Prescott, G. Algae of the Western Great Lakes Area; WM. C. Brown Co. Publishers: Dubuque, Iowa, $1962 ;$ p. 977.

38. Whitford, L.; Schumacher, G. A Manual of Freshwater Algae; Sparks Press: Raliegh, North Carolina, 1984 ; p. 324.

39. Lobeck, A.K. The Midland Trail in Kentucky, a Physiographic and Geologic Guidebook to U.S. Highway; The Kentucky Geological Survey: Frankfort, KY, USA, 1930; Volume 33, pp. 165-252.

40. Guiry, M.D.; Guiry, G.M. AlgaeBase World-Wide Electronic Publication; National University of Ireland: Galway, Ireland, 2019.

41. Love, J.; Selker, R.; Marsman, M.; Jamil, T.; Dropmann, D.; Verhagen, A.J.; Ly, A.; Gronau, Q.F.; Smira, M.; Epskamp, S.; et al. JASP: Graphical statistical software for common statistical designs. J. Stat. Softw. 2019, 88. [CrossRef]

42. Ter Braak, C.J.F.; Šmilauer, P. CANOCO Reference Manual and CanoDraw for Windows User's Guide: Software for Canonical Community Ordination (version 4.5); Microcomputer Power Press: Ithaca, NY, USA, 2002; p. 500.

43. Novakovsky, A.B. Abilities and base principles of program module "GRAPHS". Sci. Rep. Komi Sci. Cent. Ural Div. Russ. Acad. Sci. 2004, 27, 1-28.

44. Barinova, S.S.; Medvedeva, L.A.; Anissimova, O.V. Diversity of Algal Indicators in Environmental Assessment; Pilies Studio Publisher: Telaviv, Israel, 2006; p. 498. (In Russian)

45. Barinova, S.S.; Bilous, O.P.; Tsarenko, P.M. Algal Indication of Water Bodies in Ukraine: Methods and Prospects; Publishing House of Haifa University: Haifa, Israel, 2019; p. 367. (In Russian)

46. Barinova, S. Essential and practical bioindication methods and systems for the water quality assessment. Int. J. Environ. Sci. Nat. Resour. 2017, 2,1-11. [CrossRef]

47. Barinova, S. On the Classification of Water Quality from an Ecological Point of View. Int. J. Environ. Sci. Nat. Resour. 2017, 2, 1-8. [CrossRef]

48. Barinova, S.; Krassilov, V.A. Algal diversity and bio-indication of water resources in Israel. Int. J. Environ. Resour. 2012, 1, 62-72.

49. 401KAR10:031. Surface Water Standards. Available online: http://www.lrc.ky.gov/kar/401/010/031reg:htm (accessed on 12 July 2019).

50. Anonymous. Water Quality Standards Handbook-Chapter 3; Publication EPA823-B-94-005a; U.S. Environmental Protection Agency, Office of Water: Washington, DC, USA, 1994. Available online: https://apps.legislature.ky. gov/law/kar/401/010/031.pdf (accessed on 12 October 2019).

51. USEPA. National Rivers and Streams Assessment 2018/19: Field Operations Manual—Non-Wadeable; EPA-841-B-17-003b; U.S. Environmental Protection Agency, Office of Water: Washington, DC, USA, 2017; p. 158.

52. McInteer, B.B. Preliminary report of the Kentucky. Algae. Ohio J. Sci. 1930, 30, 131-142.

53. Orser, J.; Dillard, G. Analysis of the periphyton of Sloan's Crossing Pong, Mammoth Cave National Park, Kentucky. Trans. Ky. Acad. Sci. 1980, 41, 60-69. 
54. Jiyenbekov, A.; Barinova, S.; Bigaliev, A.; Nurashov, S.; Sametova, E.; Fahima, T. Algal comparative floristic of the Alakol Lake Natural State Reserve and other lakes in Kazakhstan. MOJ Ecol. Environ. Sci. 2018, 3, 252-258. [CrossRef]

55. Barinova, S. The effect of altitude on distribution of freshwater algae in continental Israel. Curr. Top. Plant Biol. 2011, 12, 89-95.

56. Barinova, S.S.; Kukhaleishvili, L.; Nevo, E.; Janelidze, Z. Diversity and ecology of algae in the Algeti National Park as a part of the Georgian system of protected areas. Turk. J. Bot. 2011, 35, 729-774. [CrossRef]

57. Barinova, S.; Liu, N.; Ding, J.Y.; An, Y.L.; Qin, X.M.; Wu, C.X. Ecological assessment of water quality of the Songhua River upper reaches by algal communities. Acta Ecol. Sin. 2016, 36, 126-132. [CrossRef]

58. Paul, M.J.; Walsh, B. Algal Indicators in Streams: A Review of their Application in Water Quality Management of Nutrient Pollution; Tetra Tech, Inc., U.S. Environmental Protection Agency, Office of Water, Office of Science and Technology: Washington, DC, USA, 2017. Available online: https://www.epa.gov/nutrient-policy-data/algalindicators-streams-review-their-application-water-quality-management (accessed on 12 October 2019).

(C) 2019 by the authors. Licensee MDPI, Basel, Switzerland. This article is an open access article distributed under the terms and conditions of the Creative Commons Attribution (CC BY) license (http://creativecommons.org/licenses/by/4.0/). 